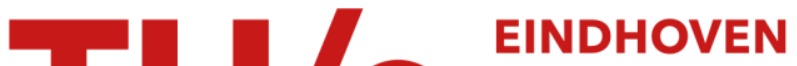 UNIVERSITY OF TECHNOLOGY
}

\section{Statistics of spatial derivates of velocity and pressure in turbulent channel flow}

Citation for published version (APA):

Vreman, A. W., \& Kuerten, J. G. M. (2014). Statistics of spatial derivates of velocity and pressure in turbulent channel flow. Physics of Fluids, 26(8), 085103-1/29. [085103]. https://doi.org/10.1063/1.4891624

DOI:

$10.1063 / 1.4891624$

Document status and date:

Published: 01/01/2014

\section{Document Version:}

Publisher's PDF, also known as Version of Record (includes final page, issue and volume numbers)

\section{Please check the document version of this publication:}

- A submitted manuscript is the version of the article upon submission and before peer-review. There can be important differences between the submitted version and the official published version of record. People interested in the research are advised to contact the author for the final version of the publication, or visit the $\mathrm{DOI}$ to the publisher's website.

- The final author version and the galley proof are versions of the publication after peer review.

- The final published version features the final layout of the paper including the volume, issue and page numbers.

Link to publication

\section{General rights}

Copyright and moral rights for the publications made accessible in the public portal are retained by the authors and/or other copyright owners and it is a condition of accessing publications that users recognise and abide by the legal requirements associated with these rights.

- Users may download and print one copy of any publication from the public portal for the purpose of private study or research.

- You may not further distribute the material or use it for any profit-making activity or commercial gain

- You may freely distribute the URL identifying the publication in the public portal.

If the publication is distributed under the terms of Article 25fa of the Dutch Copyright Act, indicated by the "Taverne" license above, please follow below link for the End User Agreement:

www.tue.nl/taverne

Take down policy

If you believe that this document breaches copyright please contact us at:

openaccess@tue.nl

providing details and we will investigate your claim. 


\title{
Statistics of spatial derivatives of velocity and pressure in turbulent channel flow
}

\author{
A. W. Vreman ${ }^{1, a)}$ and J. G. M. Kuerten ${ }^{2}$ \\ ${ }^{1}$ AkzoNobel, Research Development and Innovation, Process Technology, P.O. Box 10, \\ 7400 AA Deventer, The Netherlands \\ ${ }^{2}$ Department of Mechanical Engineering, Eindhoven University of Technology, P.O. Box 513, \\ 5600 MB Eindhoven, The Netherlands and Faculty EEMCS, University of Twente, P.O. Box \\ 217, 7500 AE Enschede, The Netherlands
}

(Received 8 April 2014; accepted 17 July 2014; published online 4 August 2014)

Statistical profiles of the first- and second-order spatial derivatives of velocity and pressure are reported for turbulent channel flow at $R e_{\tau}=590$. The statistics were extracted from a high-resolution direct numerical simulation. To quantify the anisotropic behavior of fine-scale structures, the variances of the derivatives are compared with the theoretical values for isotropic turbulence. It is shown that appropriate combinations of first- and second-order velocity derivatives lead to (directional) viscous length scales without explicit occurrence of the viscosity in the definitions. To quantify the non-Gaussian and intermittent behavior of fine-scale structures, higher-order moments and probability density functions of spatial derivatives are reported. Absolute skewnesses and flatnesses of several spatial derivatives display high peaks in the near wall region. In the logarithmic and central regions of the channel flow, all first-order derivatives appear to be significantly more intermittent than in isotropic turbulence at the same Taylor Reynolds number. Since the nine variances of first-order velocity derivatives are the distinct elements of the turbulence dissipation, the budgets of these nine variances are shown, together with the budget of the turbulence dissipation. The comparison of the budgets in the near-wall region indicates that the normal derivative of the fluctuating streamwise velocity $\left(\partial u^{\prime} / \partial y\right)$ plays a more important role than other components of the fluctuating velocity gradient. The small-scale generation term formed by triple correlations of fluctuations of first-order velocity derivatives is analyzed. A typical mechanism of small-scale generation near the wall (around $y^{+}$ $=1$ ), the intensification of positive $\partial u^{\prime} / \partial y$ by local strain fluctuation (compression in normal and stretching in spanwise direction), is illustrated and discussed. ( $) 2014$ AIP Publishing LLC. [http://dx.doi.org/10.1063/1.4891624]

\section{INTRODUCTION}

Direct numerical simulation (DNS) is a powerful tool to obtain insight in the details of incompressible turbulent channel flow. ${ }^{1,2}$ Since the first DNS of turbulent channel flow in $1987,{ }^{3}$ many direct numerical simulations of channel flow have been performed, see Refs. 4-18 for some of them. One trend in this field of research is to perform the simulations at increasingly large Reynolds number; $R e_{\tau}=4000$ has recently been passed. ${ }^{17,18}$ Another recent trend is to increase the numerical resolution without increasing the Reynolds number. ${ }^{11,12,14-16}$ For $R e_{\tau}=180$ higher resolution was shown to be essential for an accurate prediction of dissipation spectra. ${ }^{15}$

Turbulence is usually described in terms of statistics and topological structures. Both types of description are important to advance the knowledge about turbulence. For turbulent channel flows, the most common statistical quantities are the mean velocity profile, root mean square profiles of the

a)E-mail: bert.vreman@akzonobel.com 
fluctuating velocity components, and the Reynolds shear stress. Other common statistical quantities are those related to Reynolds stress budgets, which give information about the turbulence production, dissipation, transport, and redistribution of the Reynolds stresses.

Since the turbulence dissipation is expressed in first-order spatial velocity derivatives, information on the velocity gradient is important to understand the fine-scale structure of the turbulence. While in many studies the velocity gradient tensor is used to understand the topological structure of the turbulence, ${ }^{19,20}$ statistical characterization of the velocity gradient tensor is probably as important. For example, Batchelor and Townsend measured statistical moments of first- and higher-order spatial derivatives of the velocity to understand the fine-scale turbulence. ${ }^{21-23}$ For turbulent channel flow statistics of the variances of individual first-order derivatives were first studied by Antonia et al. up to $R e_{\tau}=395 .^{24}$

Several statistical moments and correlations of the velocity gradient and higher-order derivatives are required for the budget of the turbulence dissipation rate. For turbulent channel flow, this budget has been computed by Mansour et al. ${ }^{25}$ The budget of the turbulence dissipation rate is similar to the enstrophy budget, which has been estimated by Tennekes and Lumley. ${ }^{26}$ So far computations of the channel flow budgets of dissipation rate,${ }^{25}$ enstrophy, ${ }^{27,28}$ and vorticity variances ${ }^{27}$ have been performed up to Reynolds number $R e_{\tau}=395$. Recent results for the skewnesses of diagonal components of the velocity gradient tensor at $R e_{\tau}=180$ show that the skewness of the longitudinal derivative of the streamwise velocity peaks around $-1.5,{ }^{15}$ much larger (in absolute terms) than the skewness typically observed in homogeneous isotropic turbulence, ${ }^{29}$ and the scarcely available experimental values for wall-bounded flows. ${ }^{30}$ For homogeneous isotropic turbulence the statistical characteristics of spatial velocity derivatives have more elaborately been investigated: probability density functions (pdfs) and higher order moments, such as skewness and flatness, for both velocity gradient and pressure gradient have been computed. ${ }^{31-36}$

In the present paper we report statistics of the first- and second-order spatial derivatives of the velocity and pressure in turbulent channel flow at $R e_{\tau}=590$. The moderate Reynolds number allowed us to perform the DNS at enhanced resolution and for a long averaging time. Nonetheless, the Reynolds number is higher than in previous studies in which statistics of first-order derivatives and the budget of the dissipation rate were shown. ${ }^{24,25,27}$ The data files of the statistics can be downloaded at www.vremanresearch.nl.

The statistics of the spatial derivatives in turbulent channel flow are interesting, since they quantify the fine-scale structure of a canonical inhomogeneous turbulent flow. In view of the Kolmogorov theory of locally isotropic turbulence, it is relevant to investigate to which extent the turbulent motion of this flow behaves as homogeneous isotropic turbulence at increasingly fine scales (increasing order of derivatives) and increasing distance from the wall. A documentation of the turbulent statistics of individual derivatives of velocity and pressure in a turbulent solution of the Navier-Stokes equations of a well-defined case is also relevant in view of the long-standing open question of the regularity (degree of smoothness) of solutions of the Navier-Stokes equations. ${ }^{37}$

Fully new elements of the present paper are: (1) the variances of the second-order derivatives and the use of second-order derivatives in the discussion of isotropic behavior of small scales in turbulent channel flow, (2) higher-order statistics and probability density functions of first-order derivatives, including flatness profiles, which quantify the intermittency of first-order velocity and pressure derivatives in turbulent channel flow, and (3) the decomposition of the budget of the dissipation rate into the nine budgets of the variances of the first-order velocity derivatives, with particular focus on the budget of the variance of $\partial u / \partial y$ and on the triple correlations that represent generation of small scales.

The contents of the paper is as follows. In Sec. II we will give definitions, introduce the transport equations for the first-order velocity derivatives, and specify the relations for the first- and secondorder spatial derivatives in homogeneous isotropic turbulence (to be able to discuss the anisotropy of derivative tensors in channel flow). In Sec. III we will describe the numerical method and show derivative spectra to check the accuracy of the results at the resolution used in the simulation. In Sec. IV we will present results for the variances of the first- and second-order derivatives and compare them with the theoretical values in homogeneous isotropic turbulence. These variances determine the magnitudes of the different contributions to the turbulence dissipation and its destruction. The 
anisotropy of these quantities will be discussed with a focus on the center of the channel. In Sec. V we will quantify the non-Gaussianity and intermittency of the fine scales with the use of probability density functions and their characteristics (skewness and flatness). In Sec. VI we will present budgets of the dissipation rate equation and variances of the first-order derivatives of velocity, and we will present results for the 27 triple correlations that constitute the fine-scale generation term of the turbulence dissipation budget. The conclusions will be summarized in Sec. VII.

\section{DEFINITIONS AND GOVERNING EQUATIONS}

\section{A. Navier-Stokes equations}

The Navier-Stokes equations for incompressible channel flow are

$$
\begin{gathered}
u_{\alpha, \alpha}=0, \\
\partial_{t} u_{i}+u_{\alpha} u_{i, \alpha}=-p_{, i}+v \Delta u_{i}+\delta_{i 1},
\end{gathered}
$$

where $u_{i}$ denotes the velocity component in direction $x_{i}, p$ the pressure divided by the density, $v$ the kinematic viscosity, $t$ time, and $\delta_{i 1}$ is the $(i, 1)$ component of the Kronecker delta. In this entire paper the summation convention is only applied for the Greek indices $\alpha, \beta$, and $\gamma$. The spatial directions $x_{1}, x_{2}$, and $x_{3}$ refer to the streamwise, normal, and spanwise direction. Spatial differentiations are denoted by subscripts, for example, $u_{1,12}=\partial^{2} u_{1} / \partial x_{1} \partial x_{2}$, and $u_{1, \alpha \alpha}=\Delta u_{1}$. For brevity we also define $x=x_{1}, y=x_{2}, z=x_{3}, u=u_{1}, v=u_{2}, w=u_{3}$. In addition we define $u_{x}=u_{1,1}, u_{y}=u_{1,2}, u_{x x}=$ $u_{1,11}$, and so forth.

In the statistical description of a turbulent flow, it is common practice to apply the Reynolds decomposition to variables, for example, $u=\bar{u}+u^{\prime}$, where the overbar denotes the averaging operator, such that $\bar{u}$ is the mean and $u^{\prime}$ the fluctuation. The variance of $u$ is defined by $\overline{u^{\prime 2}}$ and the root mean square value, $\operatorname{Rms}(u)$, is equal to the standard deviation $\sigma_{u}=\left(\overline{u^{\prime 2}}\right)^{1 / 2}$. The standardized third and fourth central moments of the probability density function are called the skewness, $S\left(u^{\prime}\right)=$ $\overline{u^{\prime 3}} / \sigma_{u}^{3}$, and the flatness $F\left(u^{\prime}\right)=\overline{u^{\prime 4}} / \sigma_{u}^{4}$.

Invariants of a turbulent flow are quantities that are invariant to an orthogonal coordinate transformation, e.g., a rotation or a reflection. Basic invariants of the primary variables are the turbulence kinetic energy, $K=\frac{1}{2} \overline{u_{\alpha}^{\prime} u_{\alpha}^{\prime}}$, and the pressure variance, $\overline{p^{\prime 2}}$. The most basic invariants of the first-order derivatives are

$$
\begin{gathered}
A=\overline{\left|\nabla \mathbf{u}^{\prime}\right|^{2}}=\overline{u_{\alpha, \beta}^{\prime} u_{\alpha, \beta}^{\prime}}, \\
A^{p}=\overline{\left|\nabla p^{\prime}\right|^{2}}=\overline{p_{, \alpha}^{\prime} p_{, \alpha}^{\prime}},
\end{gathered}
$$

while the most basic invariants of the second-order derivatives are

$$
\begin{aligned}
B & =\overline{\left|\nabla \nabla \mathbf{u}^{\prime}\right|^{2}}=\overline{u_{\alpha, \beta \gamma}^{\prime} u_{\alpha, \beta \gamma}^{\prime}}, \\
B^{p} & =\overline{\left|\nabla \nabla p^{\prime}\right|^{2}}=\overline{p_{, \alpha \beta}^{\prime} p_{, \alpha \beta}^{\prime}} .
\end{aligned}
$$

The invariant $A$ is proportional to the turbulence dissipation of kinetic energy, while the invariant $B$ is proportional to the destruction of the turbulence dissipation. In this paper, the turbulence dissipation is defined by $\epsilon=\nu A$, like in Mansour et al. ${ }^{25}$

Alternative definitions of the turbulence dissipation are $\nu \Omega$ and $\nu \tilde{S}$, where $\Omega=\nu \overline{\omega_{\alpha}^{\prime} \omega_{\alpha}^{\prime}}$ and $\tilde{S}=2 \nu \overline{s_{\alpha \beta}^{\prime} s_{\alpha \beta}^{\prime}}$, based on the vorticity vector $\omega=\nabla \times \mathbf{u}$ and strain rate tensor $s=\frac{1}{2}\left(\nabla \mathbf{u}+(\nabla \mathbf{u})^{T}\right)$, respectively. It is straightforward to derive $\Omega=A-Y$ and $\tilde{S}=A+Y$ with $Y=\left(\overline{u_{\alpha}^{\prime} u_{\beta}^{\prime}}\right)_{, \alpha \beta}$. This implies that $A$ is precisely the average of $\Omega$ and $\tilde{S}$. In addition, the structure of $Y$ implies that $A, \Omega$, and $\tilde{S}$ are identical in homogeneous turbulence. In inhomogeneous turbulence the three quantities are not the same, but the differences are usually small. ${ }^{20,24,26,38}$ In the present channel flow, $|Y| / A$ is about 0.01 at the center, and the maximum of this ratio is about 0.04 , attained around $y^{+}=30$. 
To investigate the invariants defined above in more detail, it is interesting to consider the composition of the invariants. For this purpose we define,

$$
\begin{aligned}
& A_{i j}=\overline{u_{i, j}^{\prime}{ }^{2}}, A_{j}^{p}=\overline{p_{, j}^{\prime}{ }^{2}}, \\
& B_{i j k}=\overline{{u_{i, j k}^{\prime}}^{2}}, B_{j k}^{p}=\overline{p_{, j k}^{\prime}} .
\end{aligned}
$$

The transport equation of $A_{i j}$ can be derived by differentiation of Eq. (2) with respect to $x_{j}$, multiplication of the result with $2 u_{i, j}$, application of the averaging operator, and finally subtraction of the equation for $\bar{u}_{i, j}^{2}$. This leads to

$$
\partial_{t} A_{i j}+\overline{u_{\alpha}} A_{i j, \alpha}=M_{i j}^{(1)}+M_{i j}^{(2)}+M_{i j}^{(3)}+G_{i j}+\Pi_{i j}-2 v B_{i j}+T_{i j}^{(t)}+T_{i j}^{(p)}+T_{i j}^{(v)},
$$

where

$$
\begin{aligned}
M_{i j}^{(1)} & =-2 \overline{u_{i, j}^{\prime} u_{\alpha, j}^{\prime}} \bar{u}_{i, \alpha}, \\
M_{i j}^{(2)} & =-2 \overline{u_{i, j}^{\prime} u_{i, \alpha}^{\prime}} \bar{u}_{\alpha, j}, \\
M_{i j}^{(3)} & =-2 \overline{u_{i, j}^{\prime} u_{\alpha}^{\prime}} \bar{u}_{i, j \alpha}, \\
G_{i j} & =-2 \overline{u_{i, j}^{\prime} u_{\alpha, j}^{\prime} u_{i, \alpha}^{\prime}}, \\
\Pi_{i j} & =2 \overline{p_{, j}^{\prime} u_{i, i j}^{\prime}}, \\
B_{i j} & =\overline{u_{i, j \alpha}^{\prime} u_{i, j \alpha}^{\prime}}, \\
T_{i j}^{(t)} & =-\left(\overline{u_{\alpha}^{\prime} u_{i, j}^{\prime}{ }^{2}}\right)_{, \alpha}, \\
T_{i j}^{(p)} & =-2\left(\overline{p_{, j}^{\prime} u_{i, j}^{\prime}}\right)_{, i}, \\
T_{i j}^{(v)} & =v \Delta A_{i j} .
\end{aligned}
$$

The first three terms at the right-hand side represent production by the mean flow (the mean shear and the gradient of mean shear). These three terms vanish in homogeneous turbulence. The term $G_{i j}$ is also a production term. We call this term the fine-scale generation. It is intimately connected to the generation of fine scales by vortex stretching. The three components of each $G_{i j}$ are defined by

$$
G_{i j k}=-2 \overline{u_{i, j}^{\prime} u_{k, j}^{\prime} u_{i, k}^{\prime}} \text {. }
$$

The fifth term is a pressure strain type of term, $\Pi_{i j}$; via the pressure gradient, turbulence dissipation is redistributed from one velocity component to another. The sixth term is negative by definition. It is the called the destruction term, because it destructs the turbulence dissipation related to $A_{i j}$. The last three terms are transport terms, turbulence transport, transport by pressure gradient, and viscous transport, respectively. These three terms, which are in divergence form, also vanish in homogeneous turbulence.

The sum of the nine equations for $A_{i j}$ provides the equation of $A$,

$$
\partial_{t} A+\overline{u_{\alpha}} A_{, \alpha}=M^{(1)}+M^{(2)}+M^{(3)}+G-2 v B+T^{(t)}+T^{(p)}+T^{(v)},
$$

where the omission of the subscript $i j$ indicates a quantity summed over the nine combinations for $(i, j)$. After multiplication of this equation by $v$, the transport equation of turbulence dissipation found in Mansour et al. ${ }^{25}$ is recovered in somewhat different notation. It appears that $\Pi_{i j}$ appears in the equation for $A_{i j}$, while due to the incompressibility constraint, there is no such term in the equation 
for $A$. This is similar to the pressure strain term that appears in the Reynolds stress equations but not in the turbulence kinetic energy equation. For homogeneous flows the equation for $A$ reduces to

$$
\partial_{t} A=G-2 v B,
$$

and $A$ and $B$ are then equivalent to the mean enstrophy and mean palinstrophy, respectively, while $2 G$ is then equivalent to the mean generation of enstrophy by vortex stretching.

The nine equations for the variances of the first-order velocity derivatives allow interesting combinations. We will consider

$$
A^{u_{i}}=A_{i 1}+A_{i 2}+A_{i 3},
$$

which provides information about the fine scales in the velocity component $u_{i}$, while $v A^{u_{i}}$ is the well-known dissipation term in the transport equation of the Reynolds stress tensor component $\overline{u_{i}^{\prime} u_{i}^{\prime}}$. Another logical recombination is $A_{x_{j}}=A_{1 j}+A_{2 j}+A_{3 j}$, which provides information about the variation of the velocity in the $x_{j}$-direction. The pressure strain terms in the equation for $A_{x_{j}}$ vanish for each $j$.

\section{B. Isotropic relations}

As a point of reference for the inhomogeneity, it is useful to present some analytical properties of the spatial derivatives in homogeneous isotropic turbulence. In homogeneous isotropic turbulence, the average operator can be interpreted as the domain average over a sufficiently large box with periodic boundary conditions in the three spatial directions. Quantities averaged in this way do not depend on location but on time. There is no mean flow, thus $u_{i}^{\prime}=u_{i}$. The three diagonal components of $\overline{u_{i, j}^{2}}$ are equal and can be represented by $\overline{u_{1,1}^{2}}$. Also the six off-diagonal components of $\overline{u_{i, j}^{2}}$ are equal; they are represented by $\overline{u_{1,2}^{2}}$. It is well-known that $\overline{u_{1,2}^{2}}=2 \overline{u_{1,1}^{2}}$, see, for example, Batchelor, ${ }^{39}$ such that $A=15 \overline{u_{1,1}^{2}}$. Furthermore, it is evident that $\overline{p_{, 1}^{2}}=\overline{p_{, 2}^{2}}=\overline{p_{, 3}^{2}}$.

The 18 (3 times 6) second-order velocity derivatives fall apart into 4 groups. Within a group the variances are the same due to the symmetry of isotropic turbulence, such that each group can be represented by a single variance. The analytical relations between the 4 representative variances have been derived by Von Kármán and Howarth: ${ }^{40} \overline{u_{1,22}^{2}}=3 \overline{u_{1,11}^{2}}, \overline{u_{1,12}^{2}}=\frac{2}{3} \overline{u_{1,11}^{2}}$, and $\overline{u_{1,23}^{2}}=\overline{u_{1,11}^{2}}$. Summation of all the variances leads to $B=35 \overline{u_{1,11}^{2}}$, which implies

$$
\begin{aligned}
& \overline{u_{1,11}^{2}}=3(B / 105), \\
& \overline{u_{1,22}^{2}}=9(B / 105), \\
& \overline{u_{1,12}^{2}}=2(B / 105), \\
& \overline{u_{1,23}^{2}}=3(B / 105) .
\end{aligned}
$$

The derivation of these relations was not based on the Navier-Stokes equations, but on the two point correlation function $R_{i j}=\overline{u_{i}(\mathbf{x}) u_{j}(\mathbf{x}+\mathbf{r})}$, the rotational and reflection symmetry of homogeneous isotropic turbulence, and on the incompressibility constraint.

To derive similar relations for the second-order derivatives of the pressure, we define the correlation function $Q(\mathbf{r})=\overline{p(\mathbf{x}) p(\mathbf{x}+\mathbf{r})}$. For symmetry reasons $Q(\mathbf{r})=\overline{p^{2}} q(r)$, where $q$ is an even function of $r\left(r^{2}=|\mathbf{r}|^{2}=r_{1}^{2}+r_{2}^{2}+r_{3}^{2}\right)$. Without loss of generality, the mean pressure is set to zero. The variance of the second-order derivative $p_{, i j}$ is rewritten as

$$
\overline{p_{, i j}^{2}}=\overline{p p_{, i i j j}}=\left(\frac{\partial^{4} Q}{\partial r_{i}^{2} \partial r_{j}^{2}}\right)_{r=0}
$$


To compute the derivatives of $Q=\overline{p^{2}} q$, we use the Taylor expansion $q=q_{0}+q_{2} r^{2}+q_{4} r^{4}+O\left(r^{6}\right)$ and obtain

$$
\begin{aligned}
& \overline{p_{, 11}^{2}}=\left(\frac{\partial^{4} Q}{\partial r_{1}^{4}}\right)_{r=0}=24 q_{4} \overline{p^{2}}, \\
& \overline{p_{, 12}^{2}}=\left(\frac{\partial^{4} Q}{\partial r_{1}^{2} \partial r_{2}^{2}}\right)_{r=0}=8 q_{4} \overline{p^{2}}
\end{aligned}
$$

Thus $\overline{p_{, 11}^{2}}=3 \overline{p_{, 12}^{2}}$.

Finally, we specify the 27 distinct contributions to the fine-scale generation term $G$ in isotropic turbulence. They can be deduced from the third-order derivatives of the triple correlation function $\overline{u_{i}(\mathbf{x}) u_{j}(\mathbf{x}) u_{k}(\mathbf{x}+\mathbf{r})}$, since algebraic manipulations allow each component $G_{i j k}$ to be expressed as a linear combination of the forms $\overline{u_{i} u_{j} u_{k, l m n}}$. The triple correlation function can be written in terms of $r_{1}, r_{2}, r_{3}$, and an odd function of $r^{39}$ Using the Taylor expansion of the latter function and algebraic manipulations, we derived the expressions for $G_{i j k}$. The 27 components fall apart into 5 groups and the results for the 5 group representatives are

$$
\begin{aligned}
& G_{111}=-2 \overline{u_{1,1}^{3}}=6(G / 105), \\
& G_{112}=-2 \overline{u_{1,1} u_{2,1} u_{1,2}}=\frac{1}{2}(G / 105), \\
& G_{121}=-2 \overline{u_{1,2}^{2} u_{1,1}}=4(G / 105), \\
& G_{122}=-2 \overline{u_{1,2}^{2} u_{2,2}}=4(G / 105), \\
& G_{123}=-2 \overline{u_{1,2} u_{3,2} u_{1,3}}=6(G / 105) .
\end{aligned}
$$

An alternative procedure to derive these relations has been described by Pope (pp. 205-206). ${ }^{41}$

\section{NUMERICAL METHOD AND VALIDATION}

We simulated incompressible turbulence channel flow at $R e_{\tau}=590$ in the computational domain $[0,2 \pi H] \times[0,2 H] \times[0, \pi H]$ with a constant streamwise forcing ( $H$ is the channel half-width). This size of the domain is the same as in Ref. 5. It is sufficiently large for our purpose, since spatial derivatives peak at relatively small scales. The Reynolds number $R e_{\tau}$ is defined by $u_{\tau} H / \nu$, where $u_{\tau}$ by definition is the wall value of $(v d \bar{u} / d y)^{1 / 2}$. Furthermore, we define $x^{+}=x / \delta_{v}, y^{+}=y / \delta_{v}$, and $z^{+}$ $=z / \delta_{v}$, where $\delta_{v}=v / u_{\tau}=H / R e_{\tau}$ is the viscous length scale very close to the wall.

The numerical method is fully spectral, Fourier in streamwise and spanwise, and Chebyshev tau in the normal direction. The method, based on the equations for the normal vorticity and $\Delta v$, is an independent implementation of the method described by Kim, Moin, and Moser. ${ }^{3}$ This implementation was previously used for simulations at different $R e_{\tau} \cdot{ }^{15,42,43}$ The time integration scheme is a three-stage Runge-Kutta method with implicit treatment of the viscous terms. ${ }^{5,44}$

The number of spectral modes used for the simulation at $R e_{\tau}=590$ was $768 \times 385 \times 768$. Since the method is dealiased with the 3/2-rule in the periodic directions, the velocity, pressure, and their derivatives were in physical space available on a grid with $1152 \times 385 \times 1152$ points. After a transient period, statistics were collected during a relatively long period of $100 H / u_{\tau}(800000$ time steps). The statistics were based on 3200 instantaneous fields (one field per 250 time steps); for statistical profiles the averaging was performed over the homogeneous directions ( $x$ and $z$ ), time, and the two channel halves. For each probability density function 2001 bins were used. The uniform bin size was 0.04 times the standard deviation of the corresponding variable. This case is listed as Case 1 in Table I. To address the effect of statistical sample size statistics were also obtained for a shorter period $40 H / u_{\tau}$ (Case 2). To address the effect of Reynolds number, post-processing software developed for the present simulation at $R e_{\tau}=590$ was applied to 1600 stored fields from a recent 
TABLE I. Numerical parameters: $h_{1}^{+}$and $h_{3}^{+}$denote the grid size in streamwise and spanwise directions (scaled with $\delta_{v}$ ), respectively, and $h_{2, c}^{+}$the maximum grid size in the normal direction (attained at the center of the channel, scaled with $\delta_{\nu}$ ). All results shown in this paper apply to Case 1, unless mentioned otherwise.

\begin{tabular}{lcccccccc}
\hline \hline Case & $R e_{\tau}$ & Box size & Resolution & $h_{1}^{+}$ & $h_{2, c}^{+}$ & $h_{3}^{+}$ & Time step & Averaging time \\
\hline 1 & 590 & $2 \pi H \times 2 H \times \pi H$ & $768 \times 385 \times 768$ & 4.8 & 4.8 & 2.4 & $0.000125 H / u_{\tau}$ & $100 H / u_{\tau}$ \\
2 & 590 & $2 \pi H \times 2 H \times \pi H$ & $768 \times 385 \times 768$ & 4.8 & 4.8 & 2.4 & $0.000125 H / u_{\tau}$ & $40 H / u_{\tau}$ \\
3 & 180 & $4 \pi H \times 2 H \times \frac{4}{3} \pi H$ & $384 \times 193 \times 192$ & 5.9 & 2.9 & 3.9 & $0.00025 H / u_{\tau}$ & $200 H / u_{\tau}$ \\
\hline \hline
\end{tabular}

high-resolution simulation at $R e_{\tau}=180$ (Case 3). ${ }^{15}$ Unless indicated otherwise, figures and data apply to Case 1 .

The adequacy of the numerical results has been validated in several ways. First, the numerical results were compared with the standard database at $R e_{\tau}=590$, the one of Moser, Kim, and Mansour $(\mathrm{MKM}){ }^{5}$ who used $384 \times 257 \times 384$ spectral modes in their simulation. We will refer to this database as the MKM database. Good agreement with most statistics available for that simulation was found; some examples of the comparison will be shown later on. Second, the present numerical statistics were also compared with statistics averaged over a shorter interval $\left(40 H / u_{\tau}\right.$, Case 2$)$; some examples of this comparison will also be shown later on. Third, the total balances in the budgets of turbulence kinetic energy and turbulence dissipation rate were checked. The deviation of these profiles from zero is an indication of the effect of discretization errors and statistical averaging time. For the turbulence kinetic energy, the balance was less than $0.1 \%$ of the turbulence dissipation rate profile. For the turbulence dissipation rate, the balance was less than $1 \%$ of the destruction of dissipation profile, with an exception of the region very close to the wall $\left(y^{+}<0.1\right)$. These exceptions were investigated, and it was concluded that they are caused by the viscous transport term very close to the wall and do not influence the results further away from the wall. The viscous transport terms contain third-order velocity derivatives, and to capture those accurately everywhere even higher resolution in the wall-normal direction is required. Fourth, the spectra of first- and second-order derivatives were checked. These will be discussed below.

Before we present the spectra, we discuss typical behavior of derivative spectra. Consider the one-dimensional streamwise spectrum $E_{u}\left(k_{1}\right)$ of velocity component $u\left(k_{i}\right.$ is the wavenumber in $x_{i}$-direction). The integral of the spectrum represents the kinetic energy in $u$. In a turbulent flow, such a spectrum can be approximated by an increasing function for small $k_{1}$, an exponentially decaying function for large $k_{1}$ (the viscous range), and an inertial $k_{1}^{-5 / 3}$ range in between (see, for example, Pope). ${ }^{41}$ The peak of $E_{u}\left(k_{1}\right)$ corresponds to an integral length scale, while the viscous range is characterized by the Kolmogorov length scale $\eta$. The spectrum of the $n$th order derivative of $u$ with respect to $x_{1}$ is then given by $k_{1}^{2 n} E\left(k_{1}\right)$. Thus the spectrum of the first-order derivative $u_{x}$ increases proportionally to $k_{1}^{1 / 3}$ in the inertial range so that it peaks in the viscous range. For very large $n$ the contributions from the large-scale and inertial range become negligible and the spectrum is proportional to $k_{1}^{2 n} \exp \left(-\gamma_{1} k_{1}\right)$, if we assume that $E_{u}$ decays proportionally to $\exp \left(-\gamma_{1} k_{1}\right)$ in the viscous range. Measurements indicate $\gamma_{1} \approx 5.2{ }^{45}$ After differentiation with respect to $k_{1}$ we find that the derivative spectrum peaks at $k_{1}=2 n / \gamma_{1}$. The surprising conclusion is that the peak of the $n$th order derivative moves to infinite wavenumber for $n \rightarrow \infty$. No matter how fine the grid is, there will always be a high-order derivative that cannot be resolved on a finite grid. The impossibility to numerically compute derivatives of arbitrarily high order is no problem, since for sufficiently large $n$ the $n$th order derivative peaks at much larger wavenumber than the Kolmogorov wavenumber, such that the derivative is not relevant for the physics of the turbulence. In other words, for very large $n$ the $n$th order derivative is active at scales much smaller than the scales where the turbulence dissipation is active. For infinite $n$, the former scales have a negligible contribution to the turbulence dissipation and of course also to the turbulence kinetic energy. These arguments imply that, unlike a velocity spectrum, a velocity derivative spectrum does not display a clear and Reynolds number dependent scale-separation, since both peak and tail of the derivative spectrum are in the viscous range. 
(a) $\mathrm{x}$ - and z-spectra

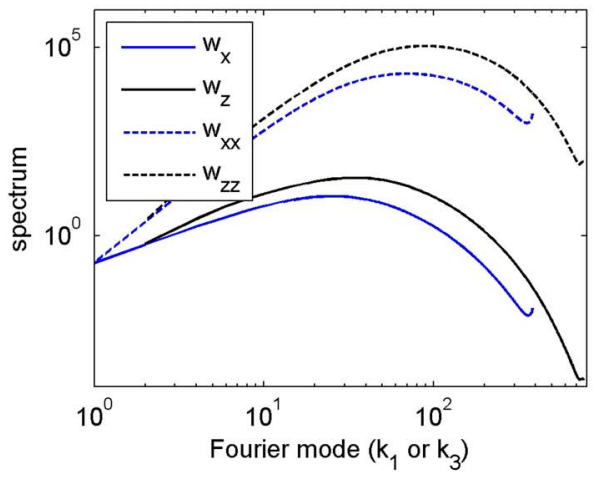

(c) fall-off $x$-spectra

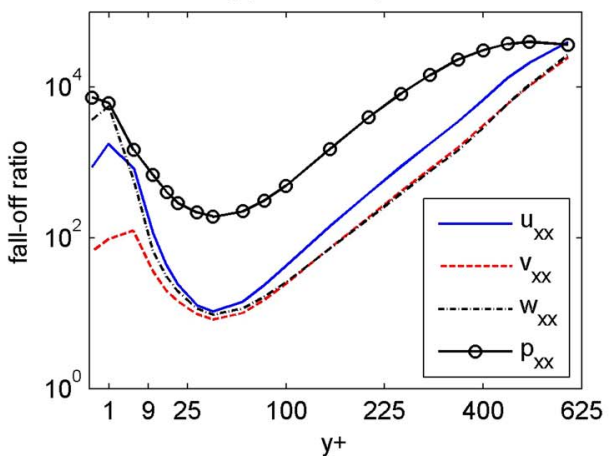

(b) $y$-spectra

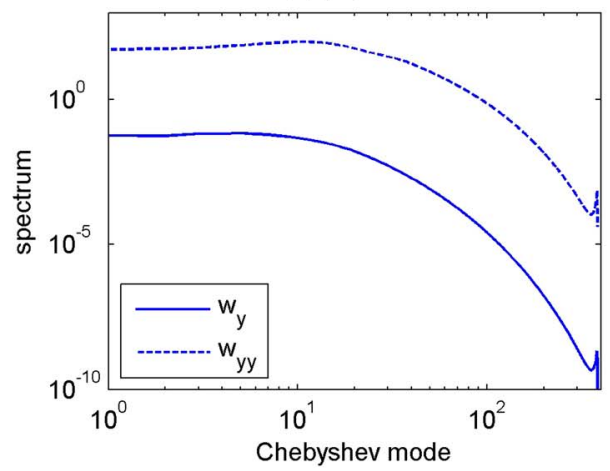

(d) fall-off z-spectra

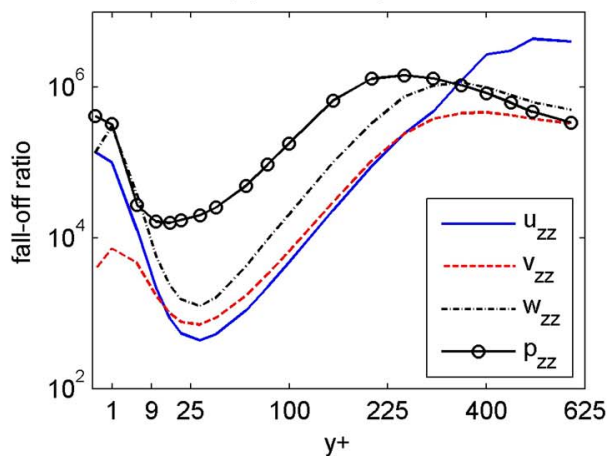

FIG. 1. (a) and (b) Examples of spectra of first- and second-order derivatives at $y^{+}=30$; (a) streamwise spectra of $w_{x}$ (solid, short curve) and $w_{x x}$ (dashed, short curve), spanwise spectra of $w_{z}$ (solid, long curve) and $w_{z z}$ (dashed, long curve); (b) Chebyshev spectra of $w_{y}$ (solid) and $w_{y y}$ (dashed). (c) and (d) Spectral fall-off (peak value of spectrum divided by tail value) of (c) streamwise and (d) spanwise spectra as function of $y^{+}$for second-order derivatives of $u$ (solid), $v$ (dashed), $w$ (dashed-dotted), and $p$ (symbols).

For this reason we consider it legitimate to verify the resolution quality of spatial derivatives by means of a spectral fall-off method (but only for spectral simulations). The spectral fall-off of a quantity is defined by the peak value of its spectrum divided by the tail value. If the spectral fall-off of a quantity is not much larger than 1, the quantity is most probably not well-resolved. Spectra of spatial derivatives and spectral fall-off profiles are shown in Fig. 1. With increase of each order of the derivative the fall-off of Fourier spectra appears to decrease with about two orders of magnitude. The comparison between Figs. 1(c) and 1(d) shows that the $z$-direction is better resolved than the $x$-direction. The roughly 100 times larger fall-off for the $z$-direction is due to the conventional choice of a grid with $h_{3}=h_{1} / 2$. Fig. 1(a) indicates that the fall-off of $x$ - and $z$-spectra of derivatives would have been comparable if we had chosen $h_{3}=h_{1}$. The lowest fall-off (about 10) is found for the second-order derivative $v_{x x}$ at $y^{+}=30$. Even in this worst case, the error in $\overline{v_{x x}^{\prime}}{ }^{2}$ was estimated to be less than $3 \%$. To obtain this error estimate, the infinite tail of the spectrum of $v_{x x}$ was modeled with a power law that matched the slope of the simulated spectrum at the wavenumber of steepest descent before the numerical cusp.

\section{VARIANCES OF SPATIAL DERIVATIVES AND ISOTROPY ANALYSIS}

In Subsection IV A we will show results for several decompositions of the invariants $2 K, A$, and $B$, including an overview of all profiles of the first- and second-order spatial derivatives of velocity and pressure. In Subsection IV B we will use these quantities to investigate the isotropy of small scales and anisotropy of larger scales in more detail. In Subsection IV C we will investigate the degree of isotropy of directional Taylor and viscous length scales. 
In channel flows one can distinguish between an inner layer with relevant length scale $\delta_{v}$ and outer layer with relevant length scale $H^{41}$ The overlap of the inner and outer layer is called the logarithmic region. Above the logarithmic region, we have the central region, and below the viscous wall region, which can be subdivided into the viscous sublayer and buffer layer. We define the lower and upper edge of the logarithmic region at $y^{+} \approx 100$ and $y^{+} \approx 0.6 H / \delta_{v}$, respectively. ${ }^{2}$ Although we will show and discuss the logarithmic and central regions in detail, all quantities in the figures have been non-dimensionalized with the friction velocity $u_{\tau}$ and the viscous length scale $\delta_{\nu}$. Unless explicitly mentioned otherwise, the figures of profiles use square-root scaling for the distance from the wall $\left(y^{+}\right)$instead of linear or logarithmic scaling. With linear scaling or logarithmic scaling of the $y^{+}$axis, details of either the viscous wall region or the central region would be less clear.

\section{A. Variances of derivatives}

The profiles of the invariants $2 K$ and $A$ are well known, since $K$ is the turbulence kinetic energy and $2 v A$ the turbulence dissipation. For the three components $A^{u}, A^{v}$, and $A^{w}$, much information is available as well, since these quantities are proportional to the dissipation terms in the budget of the diagonal Reynolds stresses. However, statistical profiles of individual first-order spatial derivatives in turbulent channel flow have been shown only a few times, and for lower $R e_{\tau}$ than in the present paper. ${ }^{15,24}$ In the former reference the isotropy relations $\overline{u_{y}^{\prime 2}}=\overline{{u_{z}^{\prime}}^{2}}=\overline{v_{x}^{\prime 2}}=2 \overline{u_{x}^{\prime 2}}$ were also investigated and found to be reasonably well satisfied in the central region at $R e_{\tau}=395$. No profiles of individual second-order spatial derivatives of velocity or pressure could be found in the literature.

The profiles of the main invariants of first-order derivatives $\left(A\right.$ and $\left.A^{p}\right)$, and the main invariants of the second-order derivatives ( $B$ and $\left.B^{p}\right)$ are shown in Fig. 2(a), together with the profile of the
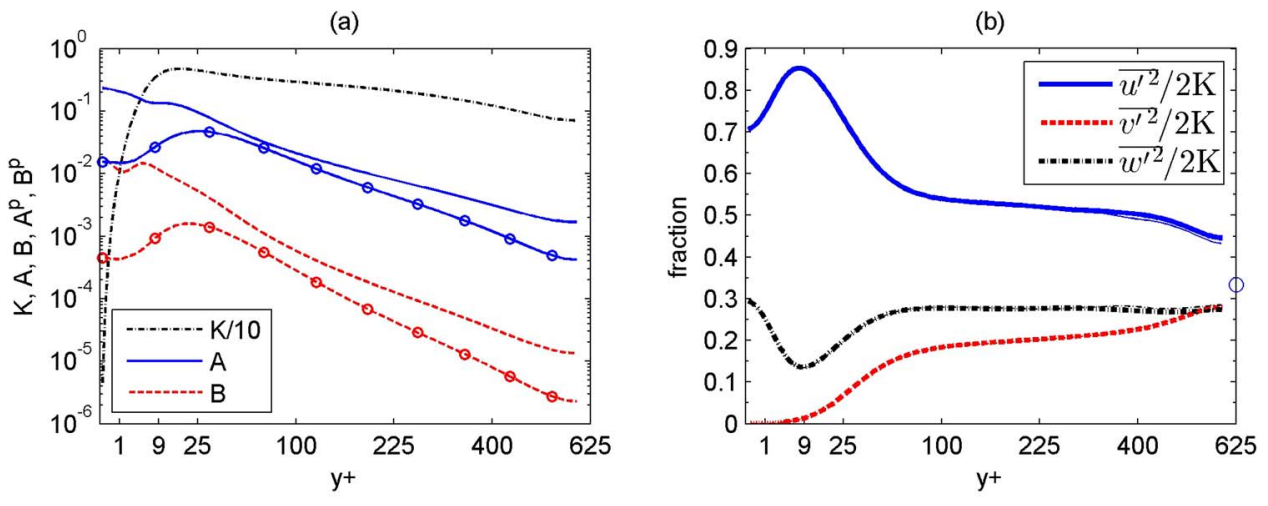

(c)
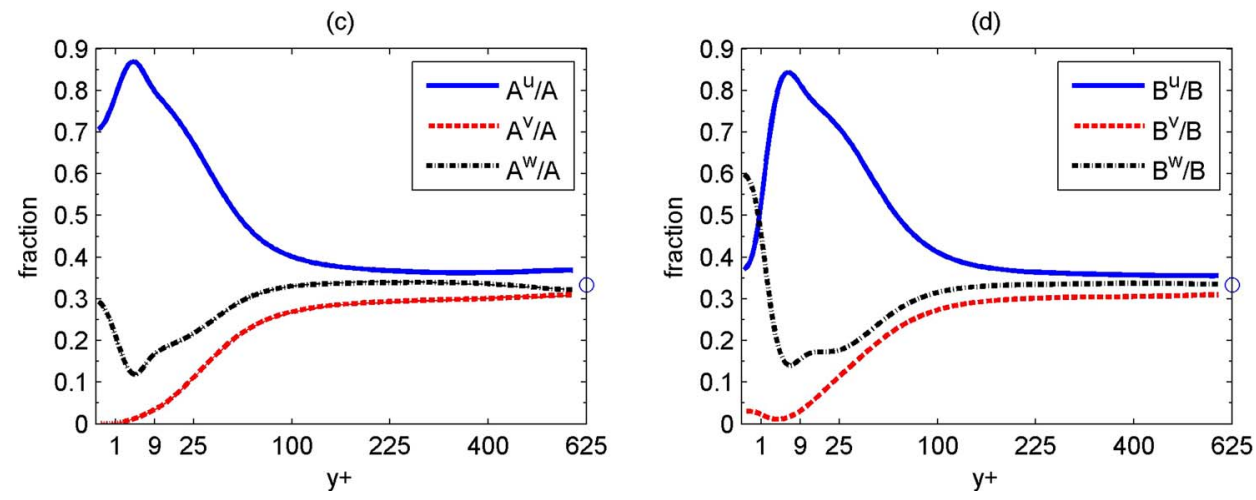

FIG. 2. (a) Invariants $K$ (divided by 10 , dashed-dotted), $A$ (solid), $B$ (dashed), $A^{p}$ (solid, circles), and $B^{p}$ (dashed, circles), all normalized with $u_{\tau}$ and $\delta_{v}$. (b)-(d) The distinct contributions from each of the three velocity components to $2 K, A$, and $B$; (b) $\overline{u^{\prime 2}} / 2 K$ (solid), $\overline{v^{\prime 2}} / 2 K$ (dashed) $\overline{w^{2}} / 2 K$ (dashed-dotted); (c) $A^{u} / A$ (solid), $A^{v} / A$ (dashed) $A^{w} / A$ (dashed-dotted); and (d) $B^{u} / B$ (solid), $B^{v} / B$ (dashed) $B^{w} / B$ (dashed-dotted). Results from the MKM database are included as thin lines in (b) and (c). The theoretical isotropic value (1/3) is indicated by a symbol (circle) in (b)-(d). 
turbulence kinetic energy $K$. In the present non-dimensionalization $v=1$, which means that the value of the turbulence dissipation is equal to $A$ and the value of the destruction of dissipation equal to $B$. At the center of the channel the quantity $A$ is about two orders of magnitudes smaller than at the wall, and $B$ even three orders of magnitude. Profiles of other quantities are therefore shown after division by (or scaling with) the profile of an appropriate reference quantity. The appropriate reference quantity is $A$ for the variances of the first-order velocity derivatives and $B$ for the variances of the second-order derivatives.

The variances of the velocity components, and those of the first-order derivatives and secondorder derivatives summed per velocity component are shown in Figs. 2(b)-2(d): $\overline{u^{\prime 2}} / 2 K$ is the fraction of energy in $u^{\prime}, A^{u} / A$ is the fraction of turbulence dissipation in $u^{\prime}$, and $B^{u} / B$ is the fraction of destruction of dissipation in $u^{\prime}$. The profiles in Figs. 2(b) and 2(c) fall almost on top of those extracted from the MKM database. ${ }^{5}$

Fig. 2(b) shows that $u^{\prime}$ has a peak contribution of about $85 \%$ to the turbulence kinetic energy in the near wall region. It is remarkable that the peak contributions of $u^{\prime}$ to the turbulence dissipation and to the destruction of turbulence dissipation are not smaller, but still about 85\% (Figs. 2(c) and 2 (d), respectively). However, in the logarithmic and central regions $\left(y^{+}>100\right),\left(A^{u}, A^{v}, A^{w}\right)$ is more isotropic than the three velocity variances, and $\left(B^{u}, B^{v}, B^{w}\right)$ is even more isotropic. Thus at increasingly fine scale the turbulence shows more isotropic behavior, which is in line with the Kolmogorov theory. The isotropy will be further discussed in Subsection IV B.

In Figs. 3(a) and 3(c) the nine distinct contributions to the turbulence dissipation are shown, expressed as the ratios $A_{i j} / A$. In the near-wall region $\left(y^{+}<20\right)$, the contribution due to the normal derivative of the streamwise velocity, $u_{y}^{\prime}$, is clearly larger than the contributions from the other first-order velocity derivatives. From these other derivatives, $u_{z}^{\prime}$ and $w_{y}^{\prime}$ give the largest contribution

(a) Contributions to $A^{4}$

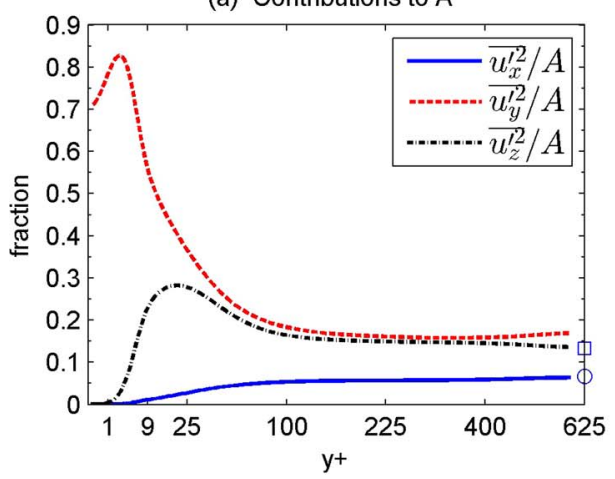

(c) Contributions to $A^{\mathrm{W}}$

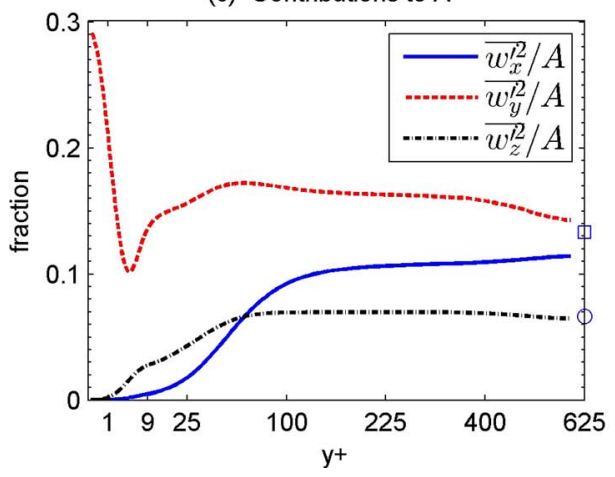

(b) Contributions to $A^{v}$

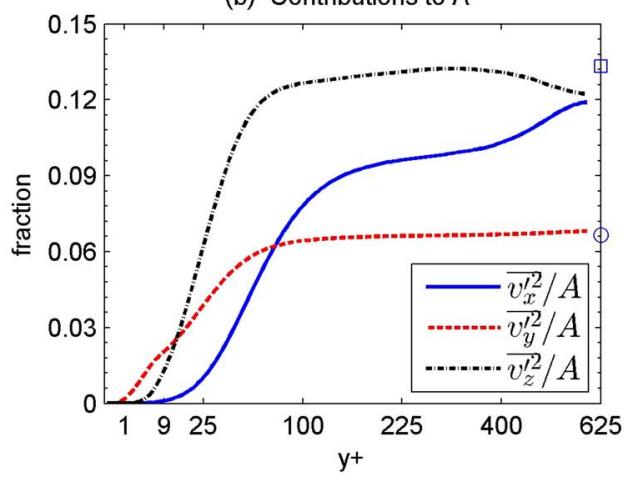

(d) Contributions to $A^{p}$

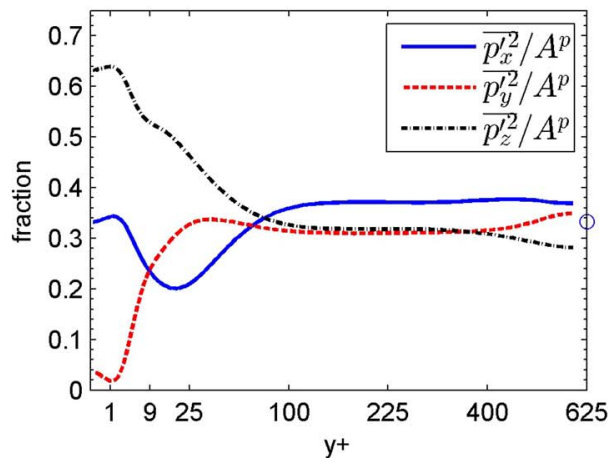

FIG. 3. Variances of first-order velocity derivatives (scaled with $A$ ) and first-order pressure derivatives (scaled with $A^{p}$ ). (a) Streamwise velocity gradient, (b) normal velocity gradient, (c) spanwise velocity gradient, and (d) pressure gradient; (a)-(d) $x$-derivative (solid), $y$-derivative (dashed), and $z$-derivative (dashed-dotted). The theoretical isotropic values are denoted by symbols: (a)-(c) 1/15 (circle) and 2/15 (square); (d) 1/3 (circle). See also Table III (Appendix). 
(a) Contributions to $\mathrm{B}^{\mathrm{U}}$

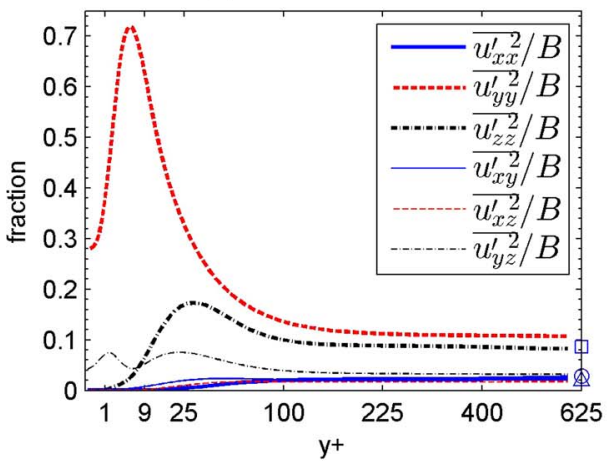

(c) Contributions to $\mathrm{B}^{\mathrm{W}}$

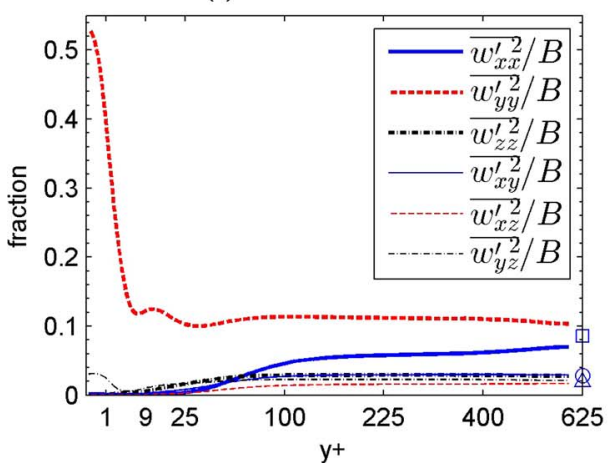

(b) Contributions to $\mathrm{B}^{\mathrm{V}}$

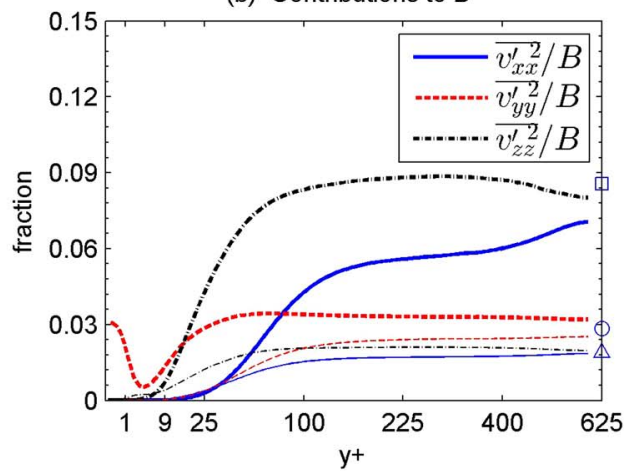

(d) Contributions to $B^{p}$

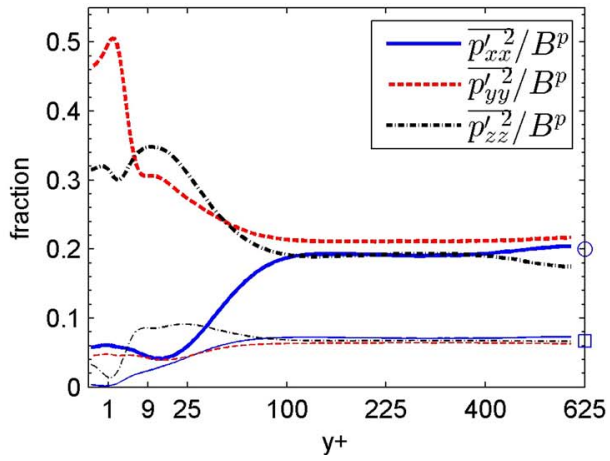

FIG. 4. Variances of second-order velocity derivatives (scaled with $B$ ) and second-order pressure derivatives (scaled with $B^{p}$ ). (a) Streamwise velocity derivatives, (b) normal velocity derivatives, (c) spanwise velocity derivatives, and (d) pressure derivatives; (a)-(d) $x x$-derivative (thick solid), $y y$-derivative (thick dashed), $z z$-derivative (thick dashed-dotted), $x y$-derivative (thin solid), $x z$-derivative (thin dashed), and $y z$-derivative (thin dashed-dotted). The theoretical isotropic values are denoted by symbols: (a)-(c) 3/105 (circle), 9/105 (square), and 2/105 (triangle); (d) 3/15 (circle) and 1/15 (square). See also Table IV (Appendix).

to the dissipation in the near wall region. The reason of the dominant contribution of $\overline{u_{y}^{\prime 2}}$ to the dissipation in the near-wall region will be analyzed in Sec. VI, where the budgets of the velocity derivative variances will be shown. The profiles of pressure gradient variances show that $p_{y}^{\prime}$ is small compared to $p_{x}^{\prime}$ and $p_{z}^{\prime}$ in the viscous sublayer, probably related to the fact that $p_{y}^{\prime}$ appears in the $v^{\prime}$ equation (near the wall $v^{\prime}$ is smaller than $u^{\prime}$ and $w^{\prime}$, because continuity implies $v_{y}^{\prime}=0$ at the wall). Furthermore, in the logarithmic and central regions, the relative variance of the $x$-derivative of the pressure converges from above to its isotropic value, in contrast to the relative variances of the three $x$-derivatives of the velocity components, which tend to be smaller than the corresponding isotropic values.

Figure 3 shows that for $y^{+}>100$ individual derivatives seem to behave more or less isotropically, although Table III in the Appendix shows that at the center the contribution of each first-order derivative to $A$ is not very close to isotropic theory. The maximum relative deviation from isotropic ratios occurs for $u_{y}^{\prime}$; at the center, $A_{12} / A$ is $26.4 \%$ larger than its isotropic value. Since approximately the same number was found in Case $2(25.6 \%)$, the deviation is not caused by statistical errors.

In Figs. 4(a)-4(c) the 24 second-order derivatives of the primary variable are shown (scaled with $B$ ). The largest peak is found for $u_{y y}^{\prime}$ at $y^{+} \approx 6$. Overall $u_{y y}^{\prime}$ is dominant among the second-order velocity derivatives in the near wall region, but surprisingly not very close to the wall; at the wall the largest contribution is not due to $u_{y y}^{\prime}$ but to $w_{y y}^{\prime}$. It is remarked that the so-called cross-derivatives of velocity (e.g., $u_{x y}^{\prime}$ ) count twice in $A$, and likewise so-called cross-derivatives of the pressure count twice in $B$. The centerline values are compared with the isotropic values derived in Sec. II and denoted by symbols in Fig. 4. Like the first-order derivatives, the second-order derivatives appear to 
TABLE II. Centerline anisotropy coefficients of the contributions of the three velocity components to $2 K, A, B, G$, and $Z$ (turbulent transport of $K$ ).

\begin{tabular}{cccccc}
\hline \hline$i$ & $3 \overline{u_{i}^{\prime 2}} / 2 K-1$ & $3 A^{u_{i}} / A-1$ & $3 B^{u_{i}} / B-1$ & $3 G^{u_{i}} / G-1$ & $3\left(\overline{Z_{i}} / Z-1\right)$ \\
\hline 1 & 0.338 & 0.106 & 0.066 & 0.187 & 0.601 \\
2 & -0.160 & -0.071 & -0.071 & -0.157 & -0.100 \\
3 & -0.178 & -0.035 & 0.005 & -0.030 & -0.501 \\
\hline \hline
\end{tabular}

behave more or less isotropically for $y^{+}>100$. Table IV in the Appendix shows the comparison at the centerline in detail. For the second-order derivatives the largest relative deviation is due to $u_{y y}^{\prime}$; $B_{122} / B$ is $24.9 \%$ larger than its isotropic value (24.4\% in Case 2 ). Although second-order derivatives correspond to smaller scales than first-order derivatives, the maximum deviation is not much smaller, probably because there are twice as many second-order than first-order derivatives.

\section{B. Anisotropy coefficients}

Investigations of isotropy of small scales in turbulent channel flow were hitherto based on the anisotropy tensor of the vorticity. ${ }^{2,24}$ In this context the Corrsin length scale is important, $L_{c}=\left(\epsilon / \bar{u}_{y}^{3}\right)^{1 / 2}$, which can be regarded as an upper limit for the isotropy of small scales. ${ }^{2,45,46}$ The underlying idea is that mean shear $\left(\bar{u}_{y}\right)$ is important for a structure if its reciprocal is smaller than the characteristic time scale of the structure. The mean shear normalized by the integral time scale $2 K / \epsilon$ is defined by $\bar{u}_{y}^{*}=2 K \bar{u}_{y} / \epsilon$ (mean shear dominates if $\bar{u}_{y}^{*} \gg 1$ ). This quantity can also be written as $\bar{u}_{y}^{*}=2\left(L / L_{c}\right)^{2 / 3}{ }^{2}$ where the integral length scale is defined by $L=K^{3 / 2} / \epsilon{ }^{41}$ In the logarithmic layer, the shear parameter $\bar{u}_{y}^{*}$ attains an approximately constant value, ${ }^{2,46}$ approximately 7 in the present case, which corresponds to $L \approx 6.5 L_{c}$ (see above).

Instead of investigating the isotropy of the three vorticity variances, the three diagonal components of the dissipation were plotted in Fig. 1. This choice has the advantage that the role of each individual velocity component remains clearly visible. In this subsection we will first discuss the anisotropic behavior shown in Fig. 1 in more detail. Second, we will perform a length scale analysis, in which statistics of individual first and second-order velocity derivatives will play an important role.

Centerline anisotropy coefficients of 5 types of variables are listed in Table II ( 0 is isotropic, theoretical extremes are -1 and 2). The variable $Z_{i}=\overline{\left(u_{i}^{\prime 2} u_{2}\right)_{, 2}}$ represents the turbulent transport term in the ${\overline{u_{i}^{\prime}}}^{2}$ equation, and $Z=Z_{1}+Z_{2}+Z_{3}$. Like Fig. 2, Table II shows that at the centerline the destruction split per velocity component is slightly more isotropic than the dissipation split per velocity component, while the latter is clearly more isotropic than the velocity variances. For all 5 variables the maximum anisotropy occurs in the $u$-quantity, also if we compare with absolute values of the anisotropies of $v$ - and $w$-quantities. With one exception, the anisotropies of $v$-and $w$-quantities shown in Table II are all negative. The implication is that at this Reynolds number both large- and fine-scale contributions to the dynamics of the $u$-velocity component are still more important than the corresponding contributions to the dynamics of either the $v$-or the $w$-velocity component.

We will discuss the details of Table II in combination with Figs. 5 and 6. In Fig. 5 the Reynolds dependency of most anisotropy coefficients in Table II is illustrated, while the dependence on $y^{+}$ of anisotropy coefficients for the $u$-quantities is shown in Fig. 6. In these figures, we do not only show our own results, but also results extracted from internet databases of spectral channel flow simulations by others. $5,7,10,18$

First we discuss the anisotropy coefficients of the velocity variances in more detail. Figs. 5(a) and 6(a) suggest that the anisotropy of the velocity variances does not converge to zero with increasing $R e_{\tau}$, at least not monotonically. The latter figure shows that at $R e_{\tau}=4200$, the anisotropy of $\overline{u^{\prime 2}}$ has developed a second peak around $y^{+} \approx 600$ (the first peak occurs in the buffer layer). This is 

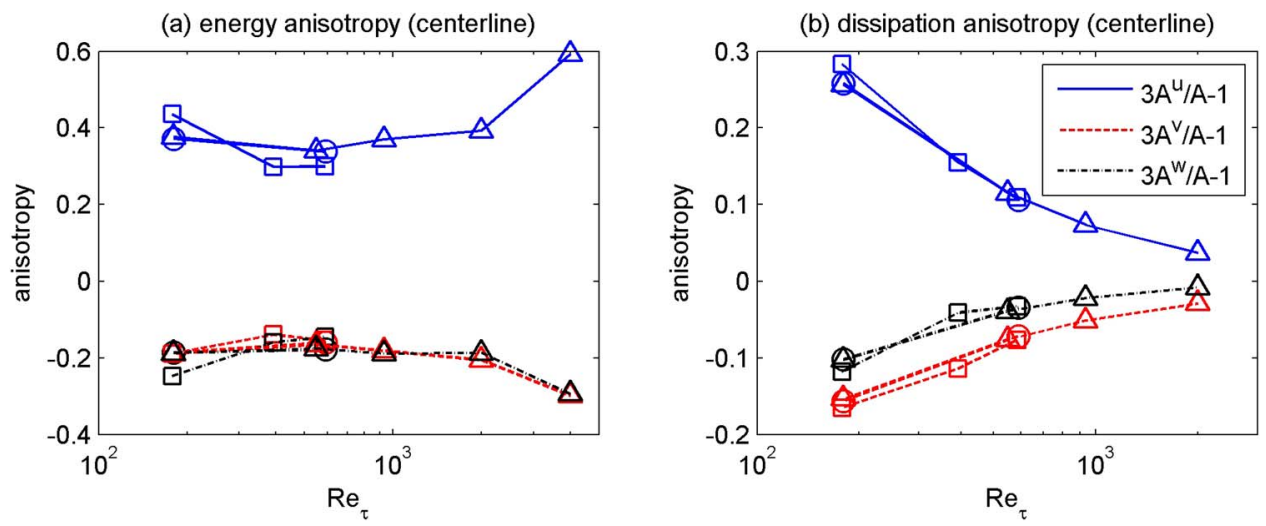

FIG. 5. Centerline anisotropies $3 \overline{u_{i}^{\prime 2}} / 2 K-1$ (a) and $3 A^{u_{i}} / A-1$ (b) as functions of $R e_{\tau}$, for $i=1$ (solid), $i=2$ (dashed), and $i=3$ (dotted). The squares denote the present Case $3\left(R e_{\tau}=180\right)$ and Case $1\left(R e_{\tau}=590\right)$, the circles databases of Moser, Kim, and Mansour $(180,395,590),{ }^{5}$ and the triangles databases of del Álamo and Jiménez $(180,550),{ }^{7}$ Hoyas and Jiménez $(950,2000),{ }^{9}, 10$ and Lozano-Durán and Jiménez (4200). ${ }^{18}$

remarkable in view of the open question whether the profile of the streamwise turbulence intensity develops a second peak at very high Reynolds number. ${ }^{1,18}$ The peak in the $u$-anisotropy observed at $R e_{\tau}=4200$ is perhaps the predecessor of a second peak in the streamwise turbulence intensity at much higher Reynolds number.

In view of the above discussion on the effect of mean shear on the upper limit of isotropic scales (the Corrsin length scale $L_{c}$ ), the question arises how the strong centerline anisotropic behavior of velocity variances can be explained. It cannot be caused by local mean shear, since $\bar{u}_{y}$ is zero there $\left(L_{c} \rightarrow \infty\right)$. We hypothesize that the large-scale anisotropy in the central region is caused by turbulent transport (turbulent diffusion); anisotropic structures are transported from the logarithmic layer, where mean shear is still dominant, to the central region, where mean shear is small. The important terms in the Reynolds stress budgets for $\overline{u_{i}^{\prime 2}}$ away from the wall are production, pressure strain, turbulent transport, and dissipation. Since the production is proportional to $\bar{u}_{i, 2}$, which is only nonzero if $i=1$, the anisotropy of the production term is maximum (except at the center, where it is not defined). Since production is small in the central region and pressure strain only acts to redistribute the energy between the components, turbulent transport becomes the dominant source of turbulence kinetic energy. The anisotropy coefficients of the three turbulent transport contributions

(a) large scale u-anisotropy

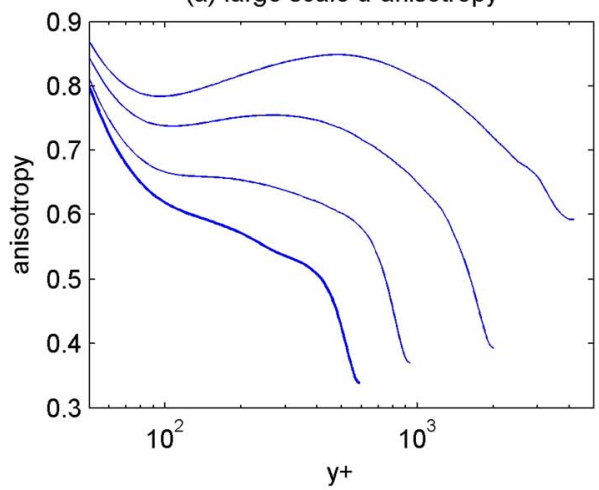

(b) fine scale u-anisotropy

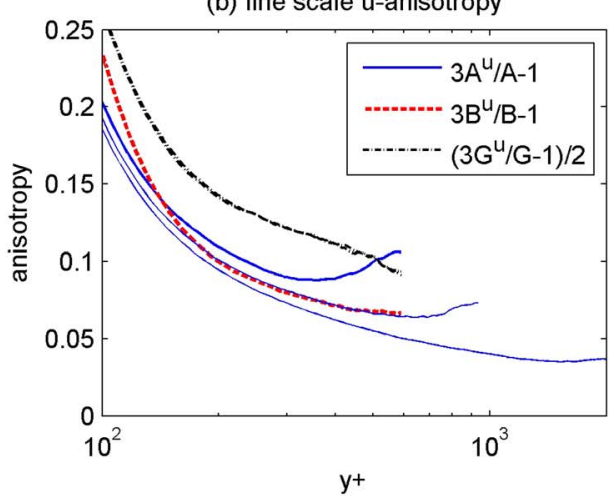

FIG. 6. (a) Anisotropy $3 \overline{u^{\prime 2}} / 2 K-1$. (b) Anisotropy $3 A^{u} / A-1$ (solid), $3 B^{u} / B-1$ (dashed), and $3 G^{u} / G-1$ divided by 2 (dashed-dotted, 2 coinciding curves, from Cases 1 and 2). (a) and (b) The thin solid lines are based on the databases of Hoyas and Jiménez $\left(R e_{\tau}=950,2000\right)^{9,10}$ and Lozano-Durán and Jiménez $\left(R e_{\tau}=4200\right) .{ }^{18}$ Logarithmic scaling of the $y^{+}$axis is used. 
have been included in Table II. The maximum of the three absolute values is larger than the maximum of the absolute anisotropy coefficients of the velocity variances. Both maxima are attained by the streamwise component.

Next, we discuss the anisotropy of fine scales per velocity component, as shown in Table II and Figs. 5 and 6. The figures show that the deviation from isotropy of the dissipation per velocity component $\left(A^{u}, A^{v}\right.$, and $\left.A^{w}\right)$ in the logarithmic and central regions becomes smaller with increasing Reynolds number. The effect of $R e_{\tau}$ on the dissipation anisotropy is relatively small for $y^{+}<200$ (including the undisplayed region $y^{+}<100$ ), but for larger $y^{+}$, the anisotropy shows a stronger dependence on $R e_{\tau}$. The anisotropy of the dissipation in the central region seems to converge to zero in the limit $R e_{\tau} \rightarrow \infty$. An interesting feature of Fig. 5(b) is that the ratio of the anisotropy of $A^{v}$ and $A^{w}$ does not show a convergence to one, unlike the ratio of the anisotropy of $\bar{v}^{\prime 2}$ and $\bar{w}^{\prime 2}$ (see Fig. 5(a)).

In addition, Table II and Fig. 6(b) show the anisotropy of the components of fine-scale quantities $B$ and $G$. Since second-order derivatives peak at smaller scale than first-order derivatives, $\left(B^{u}, B^{v}, B^{w}\right)$ are less anisotropic than $\left(A^{u}, A^{v}, A^{w}\right)$ at the centerline (Table II), and in fact for $y^{+}>$ 150 at $R e_{\tau}=590$ (Fig. 6(b)). For $150<y^{+}<300$ the anisotropy of $B^{u}$ at $R e_{\tau}=590$ is approximately as low as the anisotropy of $A^{u}$ at $R e_{\tau}=950$. In contrast to the destruction term $(2 v B)$, the fine-scale generation term $G$, which consists out of triple correlations of the fluctuations of first-order velocity derivatives, is less isotropic than the dissipation (Table II). This is the case in the logarithmic and central regions, see Fig. 6(b) (in which the anisotropy of $G^{u}$ divided by 2 is shown). This may be surprising, since why would $G$, which is more nonlinear than $A$, be more anisotropic? The anisotropy of $G^{u}$ approximately coincides for the two averaging times (Cases 1 and 2 in Fig. 6), thus the results are not a statistical error. Apparently, the stronger nonlinearity does not necessarily mean more randomness; the nonlinearity in $G$ represents vortex stretching by small scales, and perhaps vortex stretching is relatively sensitive to the larger anisotropic scales or it includes backscatter. Although the Reynolds dependency is not explicitly shown for $B$ and $G$, we found for each of the three small scale quantities $A, B$, and $G$ that the anisotropy at $R e_{\tau}=590$ is smaller than at $R e_{\tau}=180$. Although $G$ is apparently more anisotropic than $A$, we expect that, in the central region, the anisotropy of $G$ also converges to zero in the limit $R e_{\tau} \rightarrow \infty$.

\section{Length scales}

Spatial derivatives can be used to define length scales, for example, the well-known Taylor microscale, which is based on the longitudinal velocity derivative. In anisotropic flows there are three choices for the direction; therefore, a logical definition for longitudinal Taylor microscales is $\lambda_{i}=\left(\overline{u_{i}^{\prime 2}} / \overline{u_{i, i}^{\prime}}\right)^{1 / 2}$ for $i=1,2$, and 3. Since $\overline{{u_{1,1}^{\prime}}^{2}}=A / 15$ and $\overline{u_{1}^{\prime 2}}=2 K / 3$ in isotropic turbulence, a logical definition for a single Taylor microscale is $\lambda=(10 K / A)^{1 / 2}$, which is a well-known expression. ${ }^{41}$ Similarly, we can define Taylor Reynolds numbers $\left.R e_{i}={\overline{u_{i}^{\prime 2}}}^{1 / 2} \lambda_{i}\right) / v$ and an overall Taylor Reynolds number $R e_{\lambda}=\left((2 K / 3)^{1 / 2} \lambda\right) / \nu$. These length scales and Reynolds numbers are shown in Figs. 7(a) and 7(b). $R e_{\lambda}$ increases from zero at the wall to its maximum 62 at $y^{+}=250$ and then slowly decreases to 45 at the centerline. However, the directional Taylor Reynolds number $R e_{1}$ can be much larger, it peaks around 170 at $y^{+} \approx 10$.

In addition, the Corrsin length scale $L_{c}$, an upper limit for isotropic small scales, ${ }^{2}$ and integral length scale $L=K^{3 / 2} / \epsilon$ are shown in Fig. 7 ( $L$ has been divided by 6.5; for $R e_{\tau}=590$, the region where $L \approx 6.5 L_{c}$ represents the region where $\bar{u}_{y}^{*}$ is approximately constant). According to Fig. 7(a), $L_{c}$ and $\lambda$ are of the same order for $y^{+}$between 100 and 400 (roughly the logarithmic region for $R e_{\tau}$ $=590)$. The large variation between the directional Taylor length scales $\left(\lambda_{1}, \lambda_{2}\right.$ and $\left.\lambda_{3}\right)$ suggests that the flow contains anisotropic scales of the order $L_{c}$. The Corrsin scale approaches infinity at the channel center $L_{c}$, due to zero mean shear. As explained above, the anisotropy at the center, which cannot be produced by the local mean shear, is most likely caused by turbulent transport.

In the following we consider the isotropy of scales much smaller than $\lambda$ and $L_{c}$. The Kolmogorov scale $\eta=\left(v^{3} / \epsilon\right)^{1 / 4}$ is the standard length scale of the dissipative range of turbulence. To estimate the size of fine structures along each a particular direction, we wish to define directional fine 
(a) Length scales

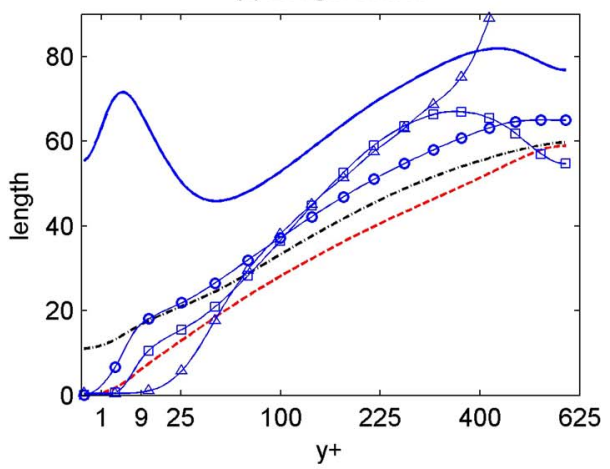

(c) Viscous length scales

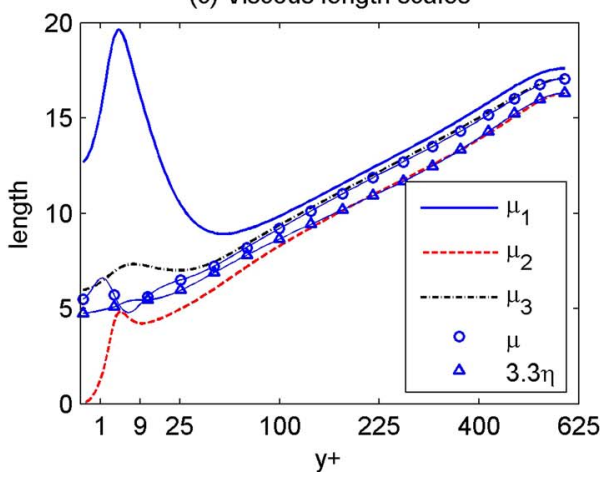

(b) Taylor Reynolds numbers

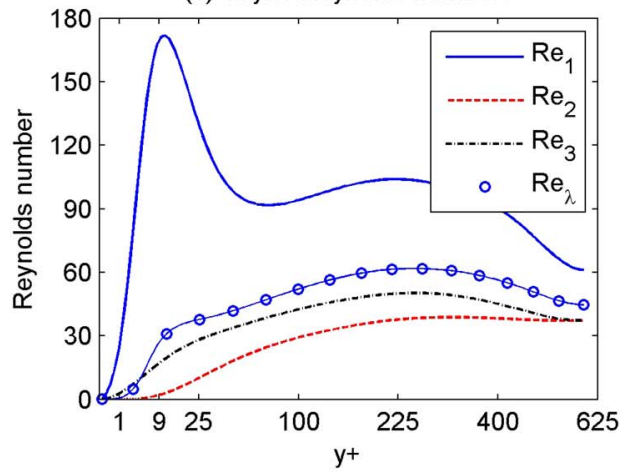

(d) Velocity scales

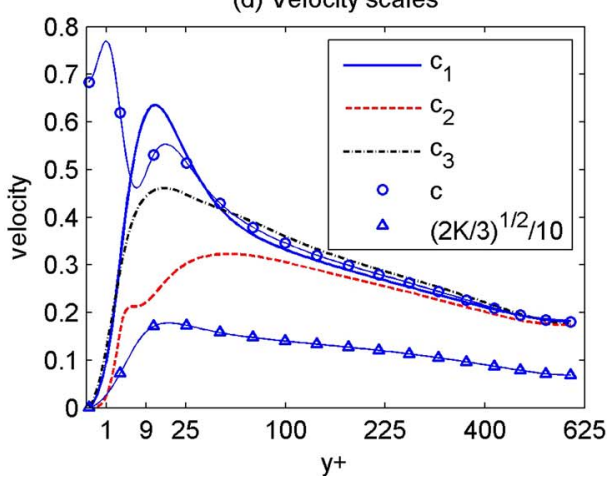

FIG. 7. (a) Taylor microscales $\lambda_{1}$ (solid), $\lambda_{2}$ (dashed), $\lambda_{3}$ (dashed-dotted), $\lambda$ (circles), Corrsin length scale $L_{c}$ (triangles), and integral length scale of turbulence $L$ divided by 6.5 (squares); (b) Taylor Reynolds numbers, $R e_{1}$ (solid), $R e_{2}$ (dashed), $R e_{3}$ (dashed-dotted), $R e_{\lambda}$ (circles); (c) length scales $\mu_{1}$ (solid), $\mu_{2}$ (dashed), $\mu_{3}$ (dashed-dotted), $\mu$ (circles), and $3.3 \eta$ (triangles); and (d) velocity scales $c_{1}$ (solid), $c_{2}$ (dashed), $c_{3}$ (dashed-dotted), and $c$ (circles), and the velocity scale $(2 K / 3)^{1 / 2}$ (divided by 10 , triangles). All length scales have been normalized with $\delta_{v}$.

scales. However, this is not straightforward. Consider, for example, the length scale definition $\tilde{\mu}_{i}=\left(v^{3} /\left(v A^{u_{i}}\right)\right)$, similar to the Kolmogorov scale, but with $\epsilon$ replaced by the dissipation of the $u_{i}$ variance. The disadvantage of this definition is that $\tilde{\mu}_{2} \rightarrow \infty$ at the wall. For this reason we define alternative length scales for fine structures by

$$
\mu_{i}=\left(\overline{u_{i, i}^{\prime}} / \overline{u_{i, i i}^{\prime}}\right)^{1 / 2},
$$

and the corresponding overall length scale

$$
\mu=((A / 15) /(3 B / 105))^{1 / 2}=(7 A / 3 B)^{1 / 2} .
$$

These length scales are shown in Fig. 7(c). They are much smaller than the Taylor microscales, which is natural since higher-order derivatives are involved. However, they are still larger than the Kolmogorov scale $\eta=\left(v^{3} / \epsilon\right)^{1 / 4}$, except $\mu_{2}$ very close to the wall. Away from the wall $\left(y^{+}>100\right)$ we observe $\mu_{i} \approx \mu$; these small length scales are clearly more isotropic than the Taylor microscales shown in Fig. 7(a). For the overall length scale $\mu$, we observe $\eta<\mu<\lambda$ in this region.

The Taylor expansion in $y$-direction of the velocity fluctuation is $u^{\prime}=a_{1} y+O\left(y^{2}\right)$ and (due to $v_{y}=0$ at the wall) $v^{\prime}=a_{2} y^{2}+O\left(y^{3}\right)$, where $y$ is the distance to the wall (the expansion of $w^{\prime}$ is similar to the expansion of $u^{\prime}$ ). The coefficients $a_{1}$ and $a_{2}$ are functions of $x, z$, and $t$ with nonzero variance. It follows that $\mu_{1}$ and $\mu_{3}$ converge to nonzero values in the near wall-limit. However, substitution of the expansion for $v^{\prime}$ in the definition of $\mu_{2}$ yields $\mu_{2}=y+O\left(y^{2}\right)$. Therefore, in the near-wall limit $\mu_{2}$ is equal to the distance to the wall, which is a physical length scale. The result $\mu_{2}$ 
$\approx y$ near the wall is also observed in Fig. 7(c) near the origin (note the root scaling of the horizontal axis).

In fact, the length scales $\mu_{i}$ and $\mu$ are viscous length scales. To support this statement with theory, we consider the three-dimensional isotropic model spectrum proposed and validated by Pope (pp. 232-238): ${ }^{41}$

$$
E(k)=C \epsilon^{2 / 3} k^{-5 / 3} f_{L}(k L) f_{\eta}(k \eta),
$$

where $E(k)$ is the kinetic energy in Fourier modes with absolute wavenumber $k$, and $C=1.5$ the Kolmogorov constant. The non-dimensional functions $f_{L}$ and $f_{\eta}$ are defined by

$$
\begin{aligned}
f_{L}(k L) & =\left(k L /\left[(k L)^{2}+c_{L}\right]^{1 / 2}\right)^{11 / 3} \\
f_{\eta}(k L) & =\exp \left(-5.2\left(\left[(k L)^{4}+c_{\eta}^{4}\right]^{1 / 4}-c_{\eta}\right)\right) .
\end{aligned}
$$

The constants $c_{L}$ and $c_{\eta}$ are determined by the requirements that the integral of $E(k)$ equals $K$ and the integral of $2 v k^{2} E(k)$ equals $\epsilon$.

For high Reynolds number, $c_{L}=6.78$ and $c_{\eta}=0.40 .{ }^{41}$ Important properties of the functions $f_{L}$ and $f_{\eta}$ are $f_{L}(k L) \rightarrow 1$ for $k \rightarrow \infty$ and $f_{\eta}(k \eta) \rightarrow 1$ for $k \rightarrow 0$. In the limit of high Reynolds number $L / \eta \rightarrow \infty$. In this limit the invariants $2 K, A$, and $B$ can be written as

$$
\begin{gathered}
2 K=2 \int_{0}^{\infty} E(k) d k=2 C \epsilon^{2 / 3} L^{2 / 3} \int_{0}^{\infty}(k L)^{-5 / 3} f_{L}(k L) d(k L), \\
A=2 \int_{0}^{\infty} k^{2} E(k) d k=2 C \epsilon^{2 / 3} \eta^{-4 / 3} \int_{0}^{\infty}(k \eta)^{1 / 3} f_{\eta}(k \eta) d(k \eta), \\
B=2 \int_{0}^{\infty} k^{4} E(k) d k=2 C \epsilon^{2 / 3} \eta^{-10 / 3} \int_{0}^{\infty}(k \eta)^{7 / 3} f_{\eta}(k \eta) d(k \eta) .
\end{gathered}
$$

The last integral in Eq. (40) is denoted by $I_{K}$, the last integral in Eq. (41) by $I_{A}$, and the last integral in Eq. (42) by $I_{B}$. We evaluated these integrals numerically and found $I_{K}=0.667, I_{A}=0.332$, and $I_{B}=0.0707$. This implies

$$
\begin{aligned}
& \lambda=(10 K / A)^{1 / 2}=\left(5 I_{K} / I_{A}\right)^{1 / 2} L^{1 / 3} \eta^{2 / 3}=3.2 L^{1 / 3} \eta^{2 / 3}, \\
& \mu=(7 A / 3 B)^{1 / 2}=\left(7 I_{A} / 3 I_{B}\right)^{1 / 2} \eta=3.3 \eta .
\end{aligned}
$$

This result does not only confirm the common knowledge that the Taylor microscale $\lambda$ is an inertial length scale (in between the integral length scale $L$ and dissipative length scale $\eta$ ), but it also shows that the small length scale $\mu$ is essentially a viscous length scale, since it is proportional to $\eta$. The relationship $\mu=3.3 \eta$ has been added to Fig. 4(c), and it appears to be a good approximation of $\mu$ in channel flow at $R e_{\tau}=590$ for $y^{+}>100$. We searched the literature for similar length scales and encountered the definition $\eta_{p}^{2}=\overline{p_{, \alpha}^{\prime} p_{, \alpha}^{\prime}} / \overline{\left(\Delta p^{\prime}\right)^{2}}$ in Ishihara et al. ${ }^{36}$ with reference to the theory of Yakhot. ${ }^{48}$ They found $\eta_{p}=3.0 \eta$ for high Reynolds number.

As discussed in Sec. III both first- and second-order derivatives peak in the viscous range. Therefore, the length scales solely based on first- and second-order derivatives are viscous length scales. Thus to define a viscous length scale it is not mandatory to include explicitly the viscosity $v$ in the definition. That length scales several times larger than $\eta$ are still viscous is in line with DNS data of isotropic turbulence, in which $\eta$ was found to lie deep into the viscous range. ${ }^{47}$

The question arises whether the small-scale velocities corresponding to the length scales based on first- and second-order velocity derivatives are indeed more isotropic than the velocity fluctuations. We therefore define small-scale velocities,

$$
c_{i}=\overline{u_{i, i}^{\prime}} /\left(\overline{{u_{i, i i}^{\prime}}^{2}}\right)^{1 / 2}
$$


and an overall small-scale velocity,

$$
c=(A / 15) /(3 B / 105)^{1 / 2}=\left(7 A^{2} / 45 B\right)^{1 / 2} .
$$

These quantities are shown in Fig. 7(d) and compared with the much larger velocity fluctuation $(2 K / 3)^{1 / 2}$, which has been divided by 10 to fit into the figure. Since all first- and second-order velocity derivatives play a role in the definition of $c, c$ is nonzero at the wall, unlike $c_{1}, c_{2}$, and $c_{3}$. The behavior of the small-scale velocities $c_{i}$ in the logarithmic and central regions $\left(y^{+}>100\right)$ is more or less isotropic, in contrast to the significant anisotropy of the velocity fluctuations $u_{i}^{\prime}$ in these regions.

\section{HIGHER ORDER MOMENTS AND PDFS}

Higher order moments and pdfs of first-order velocity and pressure derivatives have been used to characterize the small scales in isotropic turbulence. ${ }^{31-36}$ In this section we apply these tools to the spatial velocity and pressure derivatives in turbulent channel flow. It is the first time that higher order moments and pdfs of derivatives are shown for turbulent channel flow, with the exception of the diagonal derivative skewnesses $\left(S\left(u_{x}^{\prime}\right), S\left(v_{y}^{\prime}\right)\right.$, and $S\left(w_{z}^{\prime}\right)$ ), which for $R e_{\tau}=180$ were also discussed in our recent paper. ${ }^{15}$ Skewness and flatness profiles of non-derivatives (velocity and pressure) in channel flow have been shown before, ${ }^{3}$ and they can also be found in the MKM database on internet. ${ }^{5}$

Normalized higher order moments, such as skewness and flatness, are a tool to quantify the non-Gaussianity and intermittency of a quantity. The skewness of a Gaussian variable is zero and its flatness equals 3 . Non-zero skewness measures the asymmetry of a variable with respect to its mean value. If the variable has a flatness larger than 3 , it is called intermittent. A strongly intermittent signal looks dormant most of the time, interrupted with short periods of activity.

\section{A. Skewness profiles}

The skewness of velocity gradients is related to the generation of small scales via $G$, since the latter also contains triple correlations of the first-order velocity derivatives. Three terms in $G$ can be directly expressed into a skewness. These are

$$
G_{i i i}=-2 \overline{u_{i, i}^{\prime}}=-2 S\left(u_{i, i}^{\prime}\right) \overline{u_{i, i}^{\prime}} .
$$

In isotropic turbulence an exact relation between $G$ and the skewness of $u_{x}^{\prime}$ exists, ${ }^{39}$ see Eq. (30). In isotropic turbulence, $S\left(u_{x}^{\prime}\right)=S\left(u_{y}^{\prime}\right)=S\left(u_{z}^{\prime}\right) \approx-0.5,{ }^{29}$ or more precisely $-0.32 R e_{\lambda}^{0.11},{ }^{36}$ which equals -0.49 for $R e_{\lambda}=45$ (centerline of our channel). The values of these three skewnesses at the channel centerline show some deviation from $-0.49: S\left(u_{x}^{\prime}\right)=-0.45, S\left(v_{y}^{\prime}\right)=-0.71$, and $S\left(w_{z}^{\prime}\right)=-0.42$.

The skewness profiles of the first-order derivatives are shown in Fig. 8. To illustrate the effect of Reynolds number, some profiles from Case 3 have been included (one example in each subplot). The trends are similar for both Reynolds numbers. Quantitatively, some values are larger for $\operatorname{Re}_{\tau}$ $=590$, others are somewhat smaller. The skewness of the streamwise longitudinal derivative, $S\left(u_{x}^{\prime}\right)$ displays at $y^{+} \approx 30$ a strong minimum of approximately -1.5 for $\operatorname{Re}_{\tau}=180$ and -1.4 for $\operatorname{Re}_{\tau}=$ 590. This minimum is caused by local coherent structures. ${ }^{15}$ Strongly negative peaks of skewness can be observed for $v_{x}^{\prime}$ and $p_{y}^{\prime}$ in the viscous sublayer. These peaks become even stronger with increasing Reynolds number (at least up to $R e_{\tau}=590$ ).

The important derivative $u_{y}^{\prime}$ is strongly positively skewed in almost the entire channel half (large positive values of $u_{y}^{\prime}$ are more likely than large negative values of $\left.u_{y}^{\prime}\right)$. $S\left(u_{y}^{\prime}\right)$ is only negligible at the center, where it should be zero due to statistical symmetry. In the other channel half, where the mean $\bar{u}_{y}$ is negative, $S\left(u_{y}^{\prime}\right)$ is also negative. In addition, $S\left(p_{y}^{\prime}\right)$ and $S\left(p_{x}^{\prime}\right)$, which like $S\left(u_{y}^{\prime}\right)$ are zero in isotropic turbulence, show significant deviation from zero almost everywhere. The significant skewnesses of $u_{y}^{\prime}, p_{x}^{\prime}$, and $p_{y}^{\prime}$ for $y^{+}>100$ are surprising, since the variances of these fine-scale quantities appear to be more or less isotropic in the logarithmic and central regions (see Sec. IV). Future research may shed light on the behavior of these quantities at high Reynolds number. 
(a)

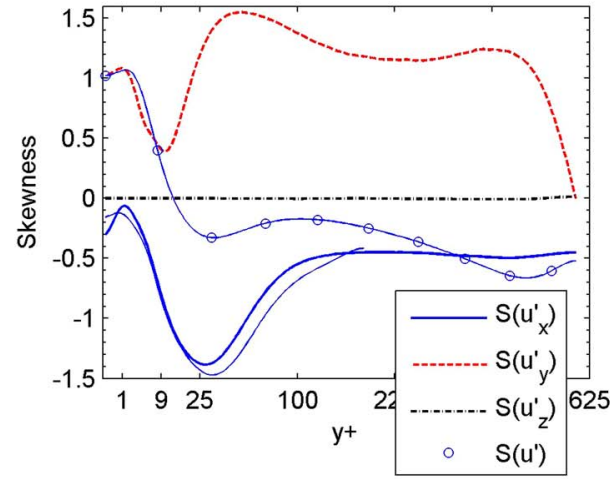

(c)

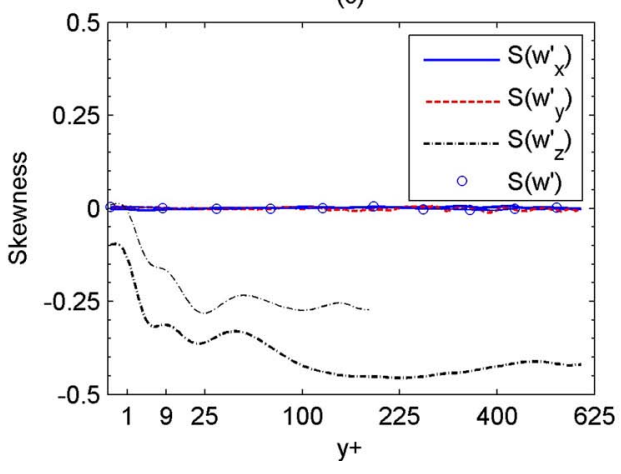

(b)

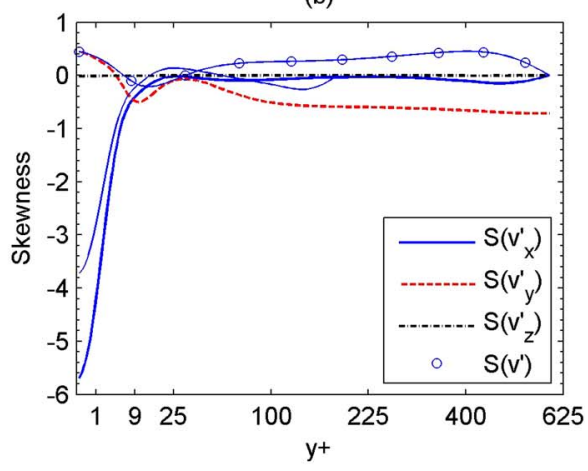

(d)

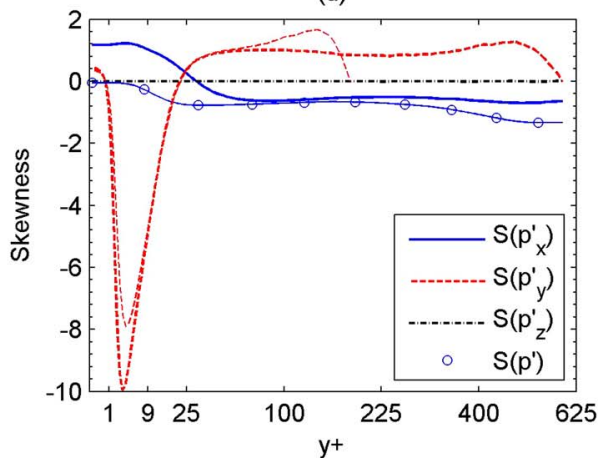

FIG. 8. Skewness of (a) gradient of $u^{\prime}$, (b) gradient of $v^{\prime}$, (c) gradient of $v^{\prime}$, and (d) gradient of $p^{\prime}$. (a)-(d) $x$-derivative (solid), $y$-derivative (dashed), and $z$-derivative (dashed-dotted). $S\left(u^{\prime}\right)$ (a), $S\left(v^{\prime}\right)(\mathrm{b}), S\left(w^{\prime}\right)$, and $S\left(p^{\prime}\right)$ are denoted by circles. Results from Case $3\left(R e_{\tau}=180\right)$ are represented by shorter thin lines (only for $u_{x}^{\prime}, v_{x}^{\prime}, w_{z}^{\prime}$, and $p_{y}^{\prime}$ ).

\section{B. Flatness profiles}

The flatness profiles of the first-order derivatives (and primary variables) are shown in Fig. 9 (square-root scaling is applied to both axes of each subplot). At most distances from the wall the derivative flatnesses are larger than the Gaussian value, which means that the derivatives are intermittent almost everywhere. Some of these flatnesses attain very large values, in particular in the near wall region. In addition, the derivative of a variable is observed to be almost everywhere more intermittent than the variable itself, which is in line with observations for isotropic turbulence. ${ }^{22}$ The large flatness of $v^{\prime}$ at the wall, which has been discussed by various authors, is related to coherent structures. ${ }^{3,14,15}$ Whereas $F\left(v^{\prime}\right)$ is high at the wall, $F\left(v_{x}^{\prime}\right)$ and $F\left(v_{z}^{\prime}\right)$ are even higher at the wall . However, the flatness of $v_{y}^{\prime}$ close to the wall is approximately the same as the flatness of $v^{\prime}$. This can be understood if we realize that near the wall $v_{y}$ is approximately equal to $v$ divided by twice the distance to the wall (which follows from the Taylor expansion of $v$ ). A few results of $R e_{\tau}=180$ have been included, $F\left(u_{x}^{\prime}\right)$ and $F\left(p_{y}^{\prime}\right)$. In the viscous sublayer, derivatives appear to be significantly more intermittent at $R e_{\tau}=590$ than at $R e_{\tau}=180$, but otherwise the intermittencies of the two cases look quite similar to each other.

In Fig. 10, the flatness values of the velocity in the logarithmic and central regions are shown. The overall Taylor Reynolds number $R e_{\lambda}$ (Fig. 7) equals 52 at $y^{+}=100$, increases to the maximum 62 at $y^{+}=250$ and then drops to 45 at $y^{+}=590$. The correlation produced by Ishihara et al. ${ }^{36}$ for the longitudinal velocity derivative in isotropic turbulence, $F\left(u_{x}^{\prime}\right)=1.14 R e_{\lambda}^{0.34}$, and other results from literature on isotropic turbulence around $R e_{\lambda}=62$ are denoted by solid symbols at $y^{+}=250$.

Fig. 10(a) shows that, like in isotropic turbulence, the flatness of an off-diagonal components of the velocity gradient is much larger than the flatness of a diagonal component (the flatness is largest for $u_{y}^{\prime}$ and $v_{x}^{\prime}$, the two derivatives of the spanwise vorticity). However, both diagonal and off-diagonal flatnesses are much larger than in isotropic turbulence at comparable $R e_{\lambda}$. Thus, the 
(a)

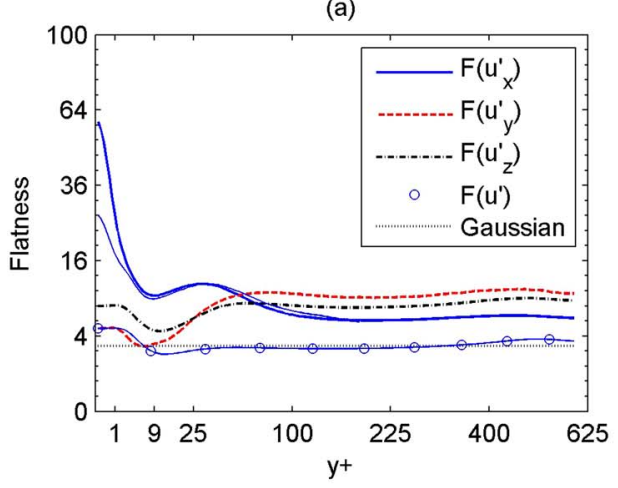

(c)

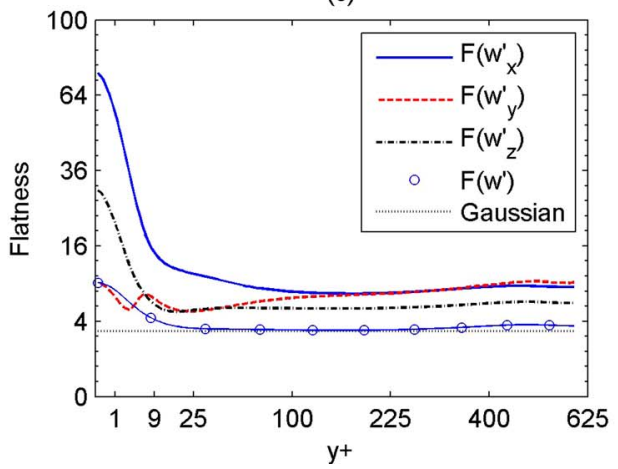

(b)

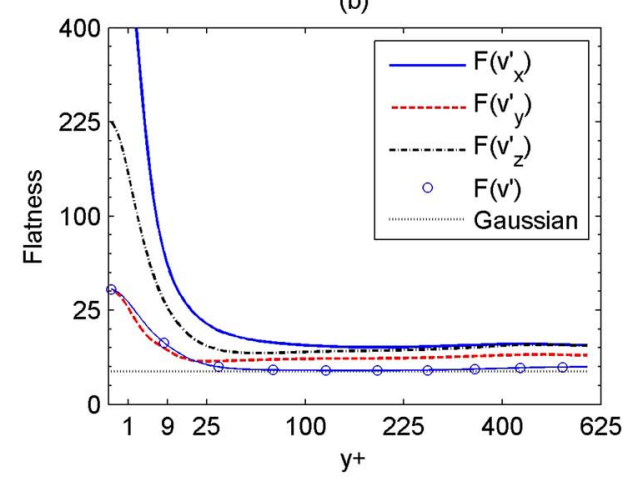

(d)

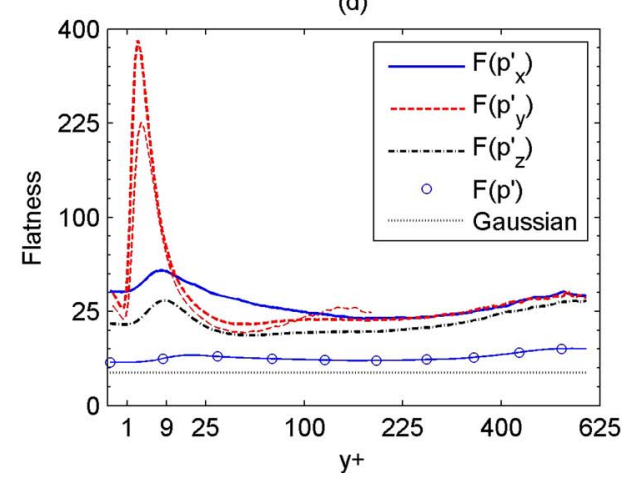

FIG. 9. Flatness profiles of (a) gradient of $u^{\prime}$, (b) gradient of $v^{\prime}$, (c) gradient of $w^{\prime}$, and (d) gradient of $p^{\prime}$. (a)-(d) $x$-derivative (solid), $y$-derivative (dashed), and $z$-derivative (dashed-dotted). $F\left(u^{\prime}\right)\left(\right.$ a), $F\left(v^{\prime}\right)(\mathrm{b}), F\left(w^{\prime}\right)$, and $F\left(p^{\prime}\right)$ are denoted by circles. Results from Case $3\left(R e_{\tau}=180\right)$ are represented by the thin lines (only for $u_{x}^{\prime}$ and $p_{y}^{\prime}$ ).

striking feature of this figure is that the fine-scale turbulence appears to be much more intermittent than in isotropic turbulence. The same applies to the pressure gradient. For the present channel, the smallest of the three centerline flatnesses of the pressure gradient $\left(F\left(p_{z}^{\prime}\right)\right)$ is equal to 27 , much larger than in isotropic turbulence, for which 8.1 for $R e_{\lambda}=38$ and 14.8 for $R e_{\lambda}=90$ were reported. ${ }^{34}$

(a) Velocity gradient flatness profiles

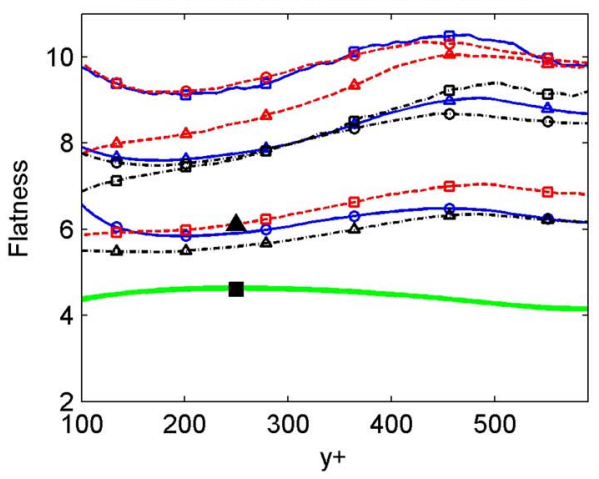

(b) Velocity and pressure flatness profiles

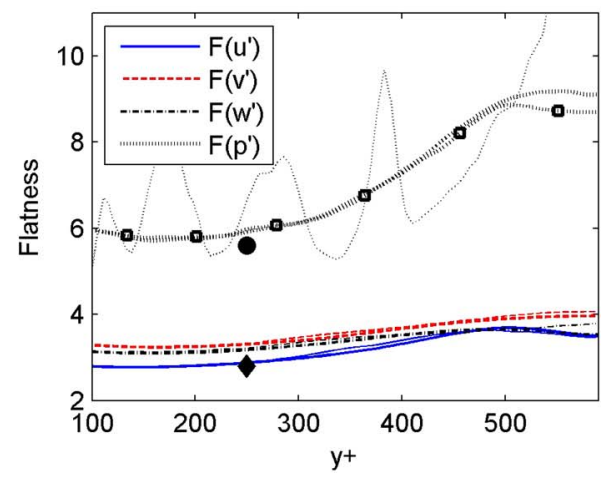

FIG. 10. Flatnesses in logarithmic and central regions (linear scaling of $y^{+}$axis). (a) $F\left(u_{x}^{\prime}\right)$ (solid, open circle), $F\left(u_{y}^{\prime}\right)$ (solid, open square), $F\left(u_{z}^{\prime}\right)$ (solid, open triangle), $F\left(v_{x}^{\prime}\right)$ (dashed, open circle), $F\left(v_{y}^{\prime}\right)$ (dashed, open square), $F\left(v_{z}^{\prime}\right)$ (dashed, open triangle), $F\left(w_{x}^{\prime}\right)$ (dashed-dotted, open circle), $F\left(w_{y}^{\prime}\right)$ (dashed-dotted, open square), and $F\left(w_{z}^{\prime}\right)$ (dashed-dotted, open triangle). Thick solid line: the correlation of $F\left(u_{x}^{\prime}\right)$ in isotropic turbulence, Ishihara et $_{\text {al. }}{ }^{36}$ (b) $F\left(u^{\prime}\right)$ (solid), $F\left(v^{\prime}\right)$ (dashed), $F\left(w^{\prime}\right)$ (dashed-dotted), $F\left(p^{\prime}\right)$ (dotted). Thick lines: present results, including one result of Case 2 (open squares). Almost coinciding curves for $F\left(p^{\prime}\right)$ represent Case 2. Thin lines: Moser, Kim, and Mansour database. ${ }^{5}$ (a) and (b) Results from DNS of isotropic turbulence: $F\left(u_{x}^{\prime}\right)$ (solid square), $F\left(u_{y}^{\prime}\right)$ (solid triangle), $F\left(u^{\prime}\right)$ (solid diamond) at $R e_{\lambda}=61$ by Jiménez et al., ${ }^{32}$ and $F\left(p^{\prime}\right)$ (solid circle) at $R e_{\lambda}=70$ by Cao et al. . $^{35}$ 
(a) pdf $u_{x}^{\prime}$

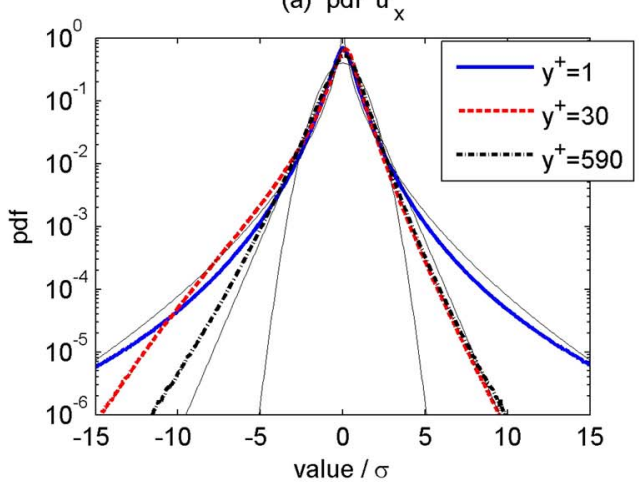

(c) pdf $u_{z}^{\prime}$

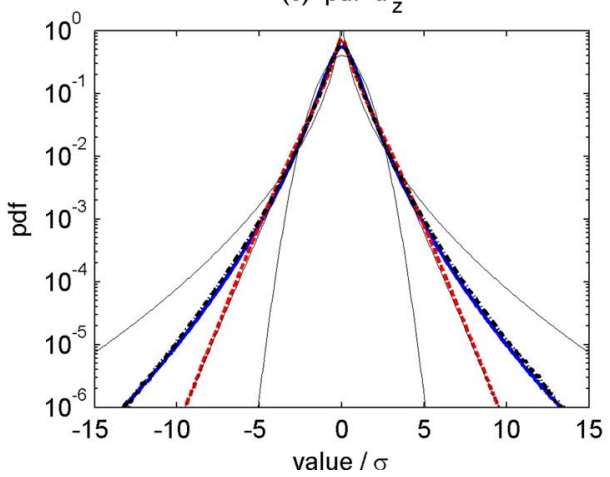

(b) pdf $u_{y}^{\prime}$

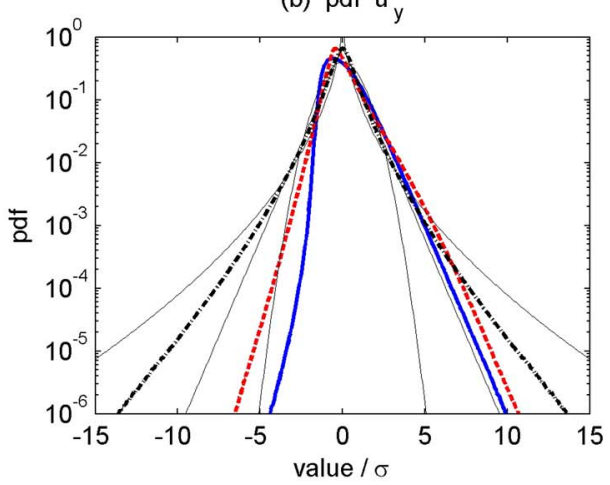

(d) pdf $\Delta p^{\prime}$

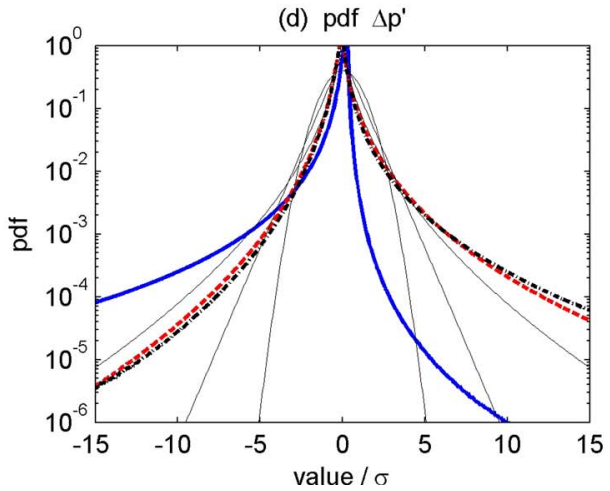

FIG. 11. Examples of probability density functions of the derivatives $u_{x}^{\prime}$ (a), $u_{y}^{\prime}$ (b), $u_{z}^{\prime}$ (c), and $\Delta p^{\prime}$ (d). (a)-(d) pdf at location $y^{+}=1$ (thick solid), $y^{+}=30$ (thick dashed), and $y^{+}=590$ (thick dash-dotted). Thin lines represent the Gaussian probability density $f_{1 / 2}$, the Laplace probability density $f_{1}$, and probability density $f_{2}$ (from narrow to wide tails).

The intermittencies of the velocity and pressure are shown in Fig. 10(b). Like in isotropic turbulence the pressure in this region is more intermittent than the three velocity components. The flatnesses of $v^{\prime}$ and $w^{\prime}$ appear to be somewhat larger than in isotropic turbulence. The flatnesses of $u^{\prime}$ and $p^{\prime}$ at $y^{+}=250$ are close to the isotropic data, but surprisingly, the pressure flatness shows a strong increase in the central region. Velocity and pressure flatness profiles from the MKM database ${ }^{5}$ on internet confirm these trends, despite large oscillations in $F\left(p^{\prime}\right)$. The statistical convergence of $F\left(p^{\prime}\right)$ in the central region is relatively slow. The maximum statistical error in our $F\left(p^{\prime}\right)$ is estimated to be about 0.5 (difference between Case 1 and Case 2), which is larger than the maximum of all absolute statistical errors of the derivative flatness profiles shown in Fig. 10(a) (about 0.2).

\section{Probability density functions}

The relatively large derivative skewnesses and flatnesses make us curious to the shape of the pdfs of spatial derivatives. The pdf of the four primary variables, the 12 first-order derivatives and the four Laplacians have been computed at 20 different locations of $y^{+}$. As an illustration Fig. 11 shows the probability density of 4 quantities at three different locations $y^{+}$. The four quantities are $u_{x}^{\prime}, u_{y}^{\prime}, u_{z}^{\prime}$, and $\Delta p^{\prime}$.

As a reference for the shape three examples of the following family of symmetric pdfs are added as thin lines,

$$
f_{n}(\xi)=b \exp \left(-a|\xi|^{1 / n}\right)
$$

where $n>0$, while the coefficients $a$ and $b$ are determined by the constraints that the standard deviation and the integral of $f_{n}$ are both 1 . Equation (48) is equivalent to the stretched exponential form in Cao et al. ${ }^{35}$ The case $n=1 / 2$ is just the standard normal distribution $N(0,1)$, which has a 

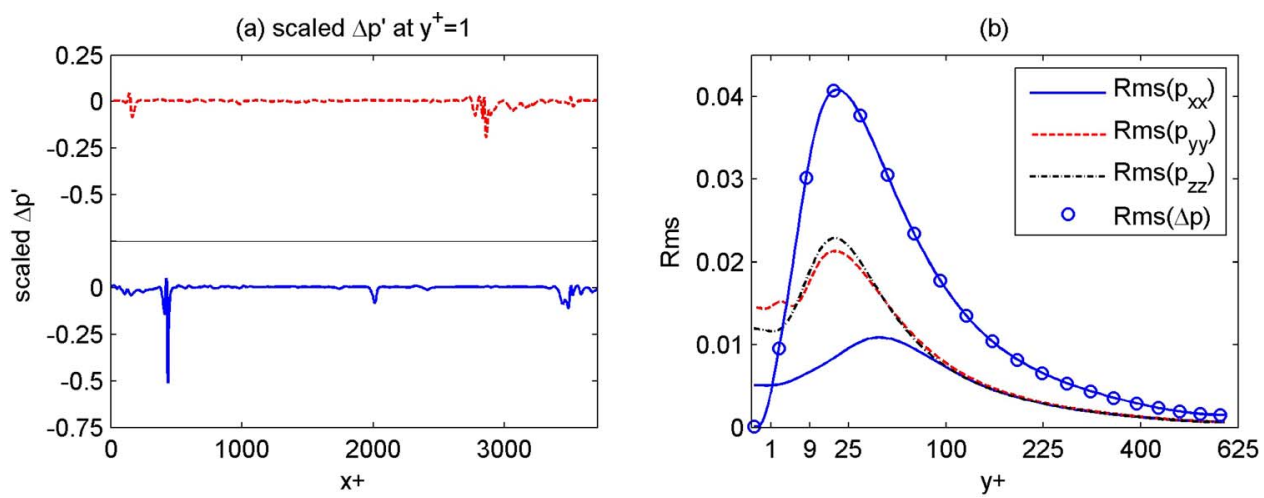

FIG. 12. (a) $\Delta p^{\prime}$ at $y^{+}=1$ and $z=0$ (dashed) and $z=L_{z} / 2$ (solid), extracted from a snapshot and scaled with the maximum of $\left|\Delta p^{\prime}\right|$ in the plane $y^{+}=1$. (b) Rms-profile of $\Delta p$ (circles) compared to the rms-profiles of its components $p_{x x}$ (solid), $p_{y y}$ (dashed), and $p_{z z}$ (dashed-dotted).

flatness of 3. The corresponding curve is parabolic in the plots, because of the logarithmic scaling of the vertical axis. The case $n=1$ is known as the two-sided exponential distribution or Laplace distribution. It has a flatness of 6 and the corresponding curve in the logarithmic plots consists of two straight lines in tent-shape. With increasing $n$, the flatness $F$ increases, the tails of the probability density function become more pronounced, while the peak at $\xi=0$ becomes sharper and the central region more narrow. For positive integer values of $n$ we derived $a^{2 n}=(3 n-1) ! /(n-1) !, b=$ $a^{n} / 2 n /(n-1) !$, and $F=(5 n-1) !(n-1) ! /((3 n-1) !)^{2}$. Thus the flatness equals $6,25.2,107.25$, 458.1 for $n=1,2,3,4$, respectively.

It is clear that the pdfs shown in Fig. 11 are strongly non-Gaussian. Some tails show exponential behavior ( $n \approx 1$, for example, for $u_{z}^{\prime}$ at $y^{+}=30$ ), but there are also tails that are better approximated by $n=2$ (for example, for $u_{x}^{\prime}$ at $y^{+}=1$ ). The positive skewness of the important $u_{y}^{\prime}$ can clearly be observed from the curves in Fig. 11(b) at $y^{+}=1$ and $y^{+}=30$.

The pdf of the Laplacian of the pressure is shown because among the computed pdfs this one takes the most unusual shapes. This quantity is strongly intermittent, and at each of the three locations one of the two tails appears to be flatter than the tails of $f_{2}$. Indeed the flatness of the Laplacian was found to be typically of the order of 100 in the logarithmic and central regions. Close to the wall, the flatness of $\Delta p^{\prime}$ becomes much larger and is accompanied with large negative skewness of $\Delta p^{\prime}$. The latter is illustrated by the very skewed shape of the pdf of $\Delta p^{\prime}$ at $y^{+}=1$. As a further illustration $\Delta p^{\prime}$ on two lines in the plane $y^{+}=1$ extracted from a snapshot is shown in Fig. 12(a). The variable is indeed very intermittent. Also the variable is clearly strongly skewed towards negative values; the absolute values of the minima are much larger than the absolute values of the maxima. This implies that the minima are more spiky than the maxima, since the mean of $\Delta p^{\prime}$ is zero by definition. Thus in the plane $y^{+}=1$ the regions with (small) positive $\Delta p^{\prime}$ cover a larger area than the regions with (large) negative $\Delta p^{\prime}$.

The remarkable behavior of $\Delta p^{\prime}$ shown in Fig. 12(a) can be explained by the presence of the wall. In incompressible channel flow $\Delta p=-u_{\alpha, \beta} u_{\beta, \alpha}$, and since $u=v=w=v_{y}=0$ at the wall, $\Delta p=0$ at the wall. Thus the no-slip condition and the continuity constraint enforce $\Delta p=0$ at the wall. Fig. 12(b) shows that, except very close to the wall, $\operatorname{Rms}(\Delta p)$ is larger than the $\operatorname{Rms}\left(p_{x x}\right)$, $\operatorname{Rms}\left(p_{y y}\right)$, and $\operatorname{Rms}\left(p_{z z}\right)$. This is expected from a quantity that is defined as the sum of $p_{x x}, p_{y y}$ and $p_{z z}$. However, near the wall $p_{x x}, p_{y y}$, and $p_{z z}$ cancel in order to comply with the implicit constraint $\Delta p=0$ at the wall. At the location considered, $y^{+}=1$, the value of $\operatorname{Rms}(p)$ is not zero yet, but has dropped below the Rms of $p_{x x}, p_{y y}$, and $p_{z z}$.

\section{BUDGETS OF FINE SCALES}

In literature the budget of dissipation rate for $R e_{\tau}=180,{ }^{25}$ and budgets of the vorticity variances up to $R e_{\tau}=395$ can be found. ${ }^{27,28}$ In this section we will consider the budget of $A$ (equivalent to the budget of the dissipation rate) for higher $\operatorname{Re}_{\tau}$ (590), and we will present budgets of individual 
(a) budget $\mathrm{A}$

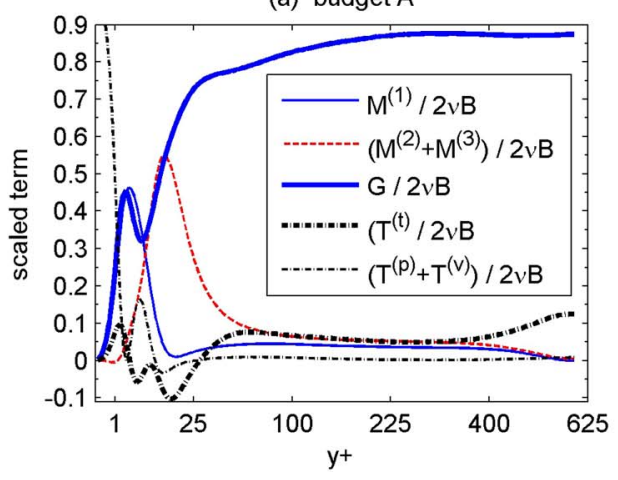

(b) generalized skewness

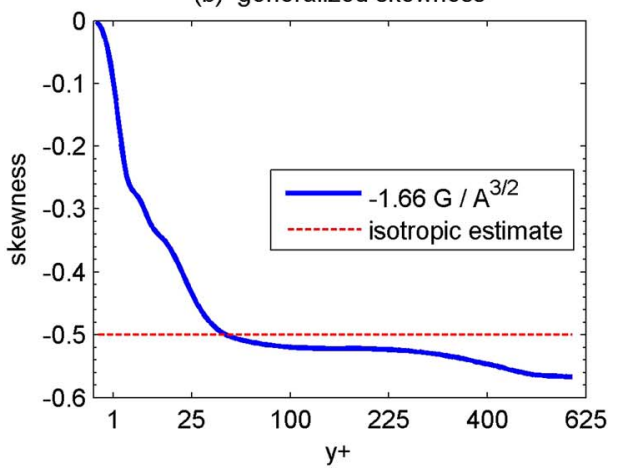

FIG. 13. (a) Terms in the budget of $A$ divided by the destruction term $2 v B: M^{(1)}$ (thin solid), $M^{(2)}+M^{(3)}$ (thin dashed), $G$ (thick solid), $T^{(t)}$ (thick dashed-dotted), $T^{(p)}+T^{(v)}$. (b) Generalized skewness $S_{G}$ (solid) and isotropic estimate -0.5 $(\text { dashed })^{29}$.

velocity gradient variances for the first time. Unlike the budgets of dissipation rate and vorticity variances, the budgets of the individual derivative variances show the role of the pressure gradient in the redistribution of fine scales (via $\Pi_{i j}$ ) and give more insight why the role of $u_{y}^{\prime}$ is different from the role of the other derivatives. In addition we will consider the distinct contributions to the small-scale generation term $G$ in the dissipation rate equation.

\section{A. Dissipation rate budget}

The budget of the transport equation for $A$ is shown in Fig. 13(a), scaled with the absolute value of the destruction term $(2 v B)$. Thus the destruction term is not shown, since $B$ was shown in Fig. 2 and after this scaling, it is simply equal to -1 . Since the term $M^{(3)}$ is the smallest one of the interactions with the mean flow (the $M$-terms in the budget), the sum of $M^{(2)}+M^{(3)}$ is shown. Likewise, since the pressure transport term $T^{(p)}$ is the smallest of all terms, the sum of pressure and viscous transport is shown.

We make the following observations. Overall, the most important terms are the generation term $G$ and destruction term $-2 v B$. Second, not only $G$, also the production terms $M^{(1)}$ and $M^{(2)}$ are important, although $M^{(2)}$ starts to become relevant somewhat further away from the wall. At the wall the viscous transport term $T^{(v)}$ is very large as expected, but Mansour et al. ${ }^{25}$ comment that this is also a somewhat artificial consequence of the common split of the total viscous effects into a diffusion term $T^{(v)}$ and a sink term $-2 v B$. Third, in the near wall region the production by mean shear $M^{(1)}$ is almost the same as $G$. Fourth, the profile of $G / 2 v B$ is nearly flat for $225<y^{+}<590$, where the generation $G$ balances about $87 \%$ of the destruction. Fifth, at the center of the channel, the remaining $13 \%$ is almost entirely caused by turbulence transport. Sixth, slightly out of the center, not only turbulence transport, but also the mean production terms contribute to the remaining $13 \%$. The first three observations, which apply to the buffer layer, were also made by Mansour et al. ${ }^{25}$ The latter three observations are new, since in the dissipation rate budget analysis by Mansour et al. only the region $y^{+}<60$ was considered.

Similar to the definition of an overall Taylor microscale based on $K$ and $A$ (see Sec. IV) it is possible to define a generalized skewness based on $G$ and $A$ by

$$
S_{G}=\left(-\frac{1}{2} G / 35\right) /(A / 15)^{3 / 2} \approx-1.66 G / A^{3 / 2},
$$

such that in isotropic turbulence $S_{G}=S\left(u_{x}^{\prime}\right)=S\left(v_{y}^{\prime}\right)=S\left(w_{z}^{\prime}\right)$. In contrast to the distinct $S\left(u_{x}^{\prime}\right)$, $S\left(v_{y}^{\prime}\right)$, and $S\left(w_{z}^{\prime}\right)$ shown in Fig. 8(a), the generalized skewness shown in Fig. 13(b) is reasonably close to the isotropic empirical value of -0.5 for $y^{+}>30$, which includes the logarithmic and central regions. 
(a) budget $A_{11}$

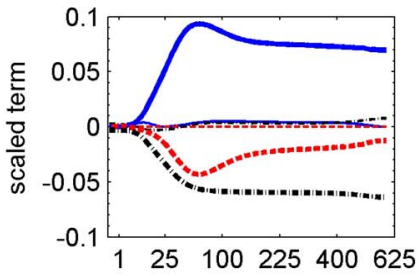

(d) budget $A_{21}$

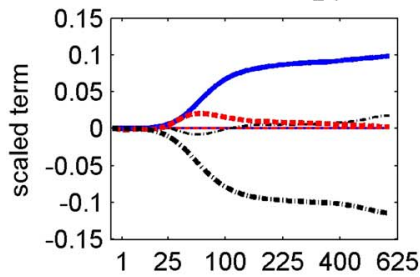

(g) budget $A_{31}$

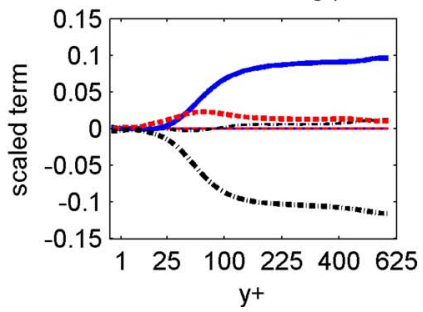

(b) budget $A_{12}$

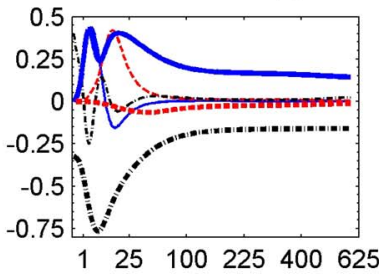

(e) budget $A_{22}$

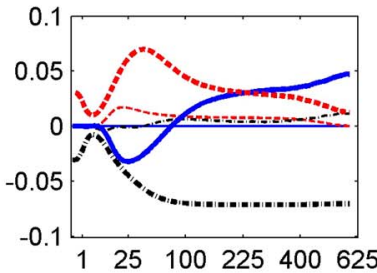

(h) budget $A_{32}$

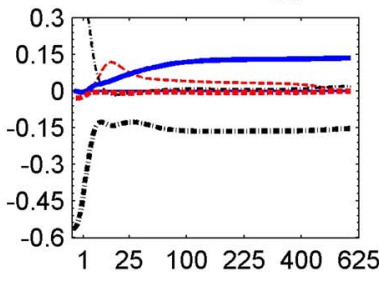

(c) budget $A_{13}$

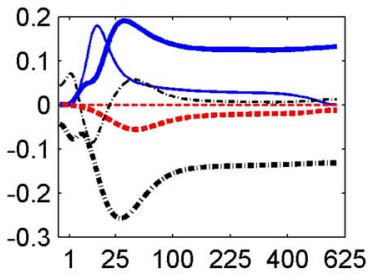

(f) budget $A_{23}$

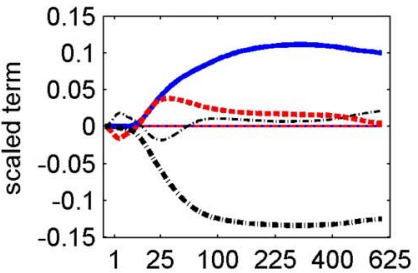

(i) budget $A_{33}$

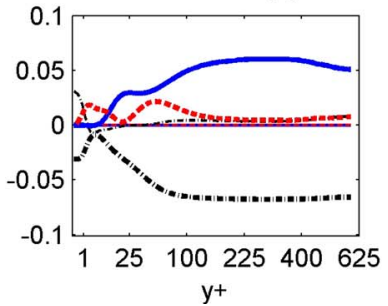

FIG. 14. (a)-(i) Terms in the budget of $A_{i j}$ divided by the destruction term $2 v B: M_{i j}^{(1)}$ (thin solid), $M_{i j}^{(2)}+M_{i j}^{(3)}$ (thin dashed), $G_{i j}$ (thick solid), $\Pi_{i j}$ (thick dashed), $-2 v B_{i j}$ (thick dashed-dotted), and $T_{i j}^{(t)}+T_{i j}^{(p)}+T_{i j}^{(v)}$ (thin dashed-dotted). Arrangement of subplots: from top to bottom $i=1,2,3$, and from left to right $j=1,2,3$.

\section{B. Budgets of variances of derivatives}

An overview of the budgets of the nine variances of the first-order velocity derivatives is shown in Fig. 14. In contrast to the zero $\Pi_{\alpha \alpha}$ in the budget of $A$, the pressure term $\Pi_{i j}$ in the budget of $A_{i j}$ is nonzero. All terms in the individual budgets have been scaled with $2 v B$. The sum of the curves reduces to the corresponding scaled terms of the total budget shown in Fig. 13(a).

We make the following observations. First, as implied by, its definition, $M_{i j}^{(1)}$ is only nonzero for $i=1$ (the equations for the variances of the gradient of $u$ ). More unexpectedly, this term is much smaller for the $x$-derivative $(j=1)$ than for the $y$ - and $z$-derivatives $(j=2$ and $j=3)$. Second, the other production by mean effects, $M_{i j}^{(2)}$ contributes to the three $y$-derivatives only $(j=2)$. The term $M_{i j}^{(3)}$, not explicitly shown, is only nonzero in the budget of the $u_{y}$ variance $(i=1$ and $j=2)$; the quantity is typically smaller than $M_{12}^{(2)}$, but it has an overall positive contribution to the generation of $u_{y}$ variance. Third, the terms $\Pi_{i j}$ show that the pressure redistributes dissipation from the derivatives of $u$ to the derivatives of $v$ and $w$, since the term is clearly negative for $i=1$, and positive for $i=2$ and $i=3$. However, the budget of $w_{y}$ variance is an exception: $\Pi_{32}$ is small and tends to be negative rather than positive (at least at the wall). Fourth, the strongest overall contribution to the destruction terms is due to destruction of $u_{y}$ variance, although at the wall itself the destruction of $w_{y}$ variance is stronger. This is in line with the discussion on the second-order velocity derivatives in Sec. IV.

The overall largest contribution to production of turbulence dissipation appears to be due to the generation term $G_{12}$, which occurs in the perhaps most interesting budget, namely, the one of $A_{12}$ (variance of $u_{y}$ ). One of the reasons that $A_{12}$ is relatively important for the turbulence dissipation is that this is the only component of $A_{i j}$ for which the four production terms, $M^{(1)}, M^{(2)}, M^{(3)}, G$, are all active (and generally positive). For the other variances only one or two of the four production terms are nonzero. 

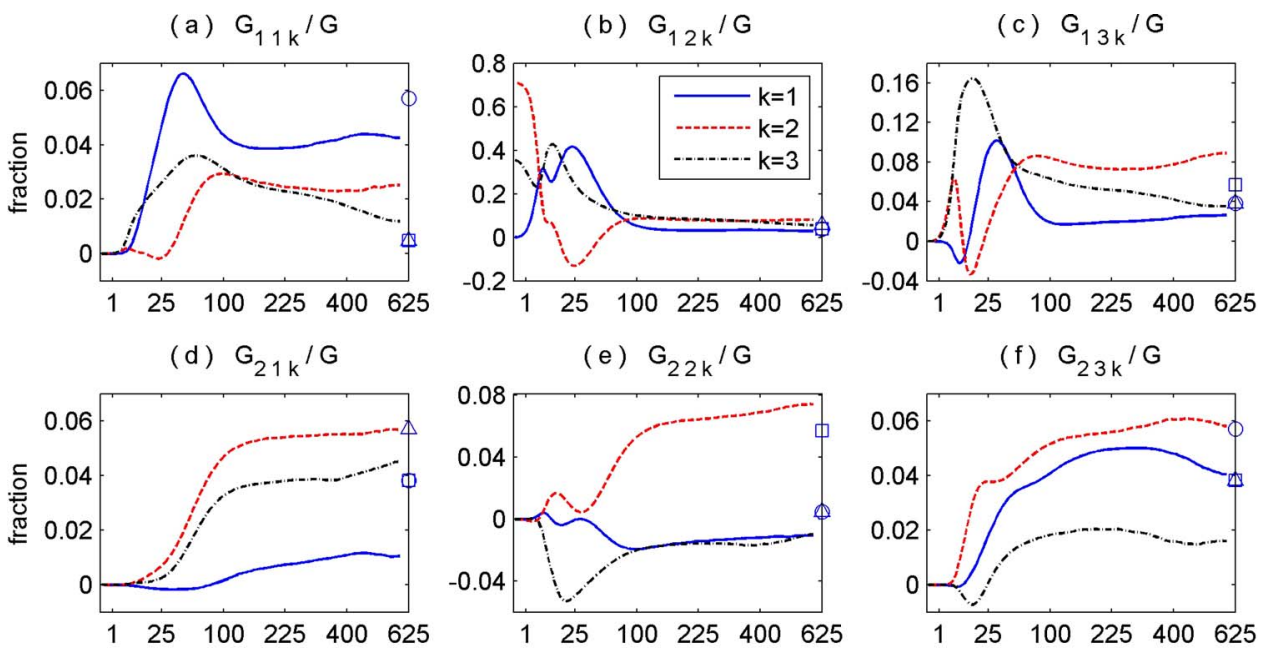

(g) $G_{31 k} / G$

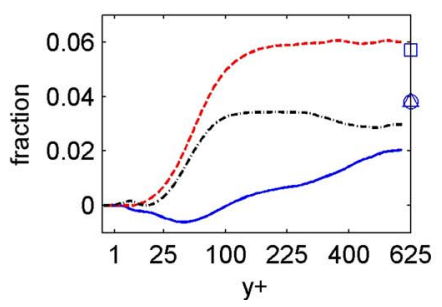

(h) $G_{32 k} / G$

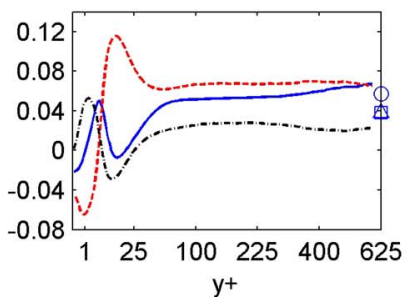

(i) $G_{33 k} / G$

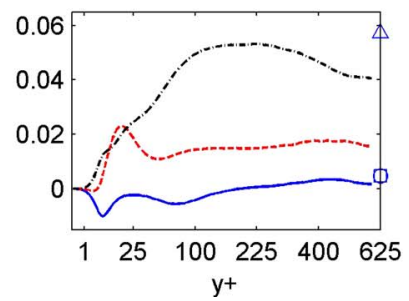

FIG. 15. (a)-(i) The contributions $G_{i j k}$ to the fine-scale generation term $G$ (scaled with $G$ ). Lines: $k=1$ (solid), $k=2$ (dashed), and $k=3$ (dashed-dotted). The theoretical isotropic values $6 / 105, \frac{1}{2} / 105$, and $4 / 105$ are denoted by symbols for $k$ $=1$ (circle), $k=2$ (square), and $k=3$ (triangle). Arrangement of subplots: from top to bottom $i=1,2,3$, and from left to right 1, 2, 3. See also Table V (Appendix).

\section{Generation of dissipation}

Since $G$ is the most important term for generation of dissipation throughout across the entire channel and is the only production term that is also nonzero in isotropic turbulence, the entire composition of $G$ is shown in Fig. 15, all 27 components, 3 for each $G_{i j}$, see also Table V. Compared to the behavior of the derivative tensors $A_{i j}$ and $B_{i j k}$ the behavior of $G_{i j k}$ is clearly less isotropic, indicating that larger scales are probably involved.

Comparing the magnitude of the relative contributions to $G$ in the region very close to the wall, the contribution of $G_{122}$ is the largest one, followed by the contribution of $G_{123}$. Both contributions appear in the budget of the $u_{y}$ variance. It is remarked that the strong anisotropy of $G_{122}$ also persists at the centerline (at least for $R e_{\tau}=590$ ), since of all $G_{i j k} / G, G_{122} / G$ shows the largest deviation from isotropic theory (see Appendix, Table V).

In order to explain the near-wall behavior, a typical snapshot of the $y^{+}=1$ plane has been investigated. We searched for the maximum of $-2 u_{1,2}^{\prime} u_{2,2}^{\prime} u_{1,2}^{\prime}=-2 u_{y}^{\prime 2} v_{y}^{\prime}$ in this plane, since the average of this term is equal to $G_{122}$. The maximum was attained at $x^{+} \approx 3300$ and $z^{+} \approx 1245$; this location is called point $Y$. Although $G_{121}$ is relatively small around $Y$, we show contours of the sum $-2 u_{y}^{\prime 2}\left(u_{x}^{\prime}+v_{y}^{\prime}\right)$ in Fig. 16(a); the average of this term is $G_{121}+G_{122}$. It is remarked that $G_{121}+G_{122}$ can also be written as $2 \overline{u_{y}^{\prime 2} w_{z}^{\prime}}$, because $u_{x}^{\prime}+v_{y}^{\prime}=-w_{z}^{\prime}$. Fig. 16(a) shows that not only the maximum of the generation $-2 u_{y}^{\prime 2}\left(u_{x}^{\prime}+v_{y}^{\prime}\right)$, but also $u_{y}^{\prime}$ itself has a local maximum near $Y$. In addition Figs. 16(c) and 16(d) show that $u_{x}^{\prime} \approx 0$ at $Y$, while $v_{y}^{\prime}$ is negative at $Y$ and close to a local minimum; continuity implies that $w_{z}^{\prime}$ is positive at $Y$.

Thus the picture of fine-scale generation illustrated by Fig. 16 is as follows, in a region of strong $u_{y}^{\prime}$ (locally intense shear layer of streamwise velocity), $u_{y}^{\prime}$ is generated by the interaction of $u_{y}^{\prime}$ with the local strain field. The local strain field compresses the shear layer in $y$-direction $v_{y}^{\prime}<0$ and 

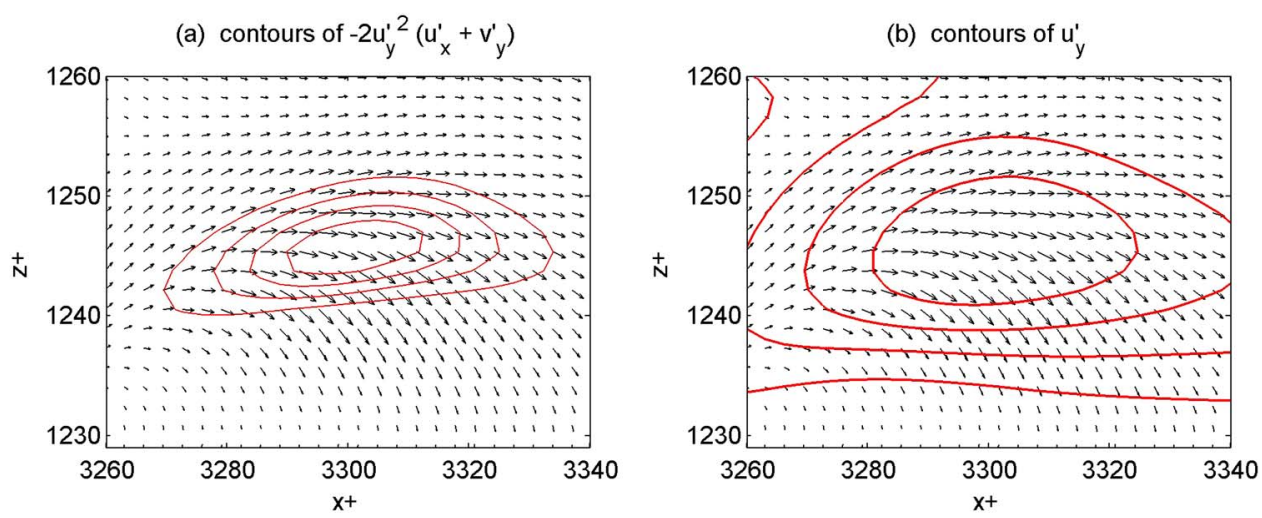

(c) contours of $u_{x}^{\prime}$

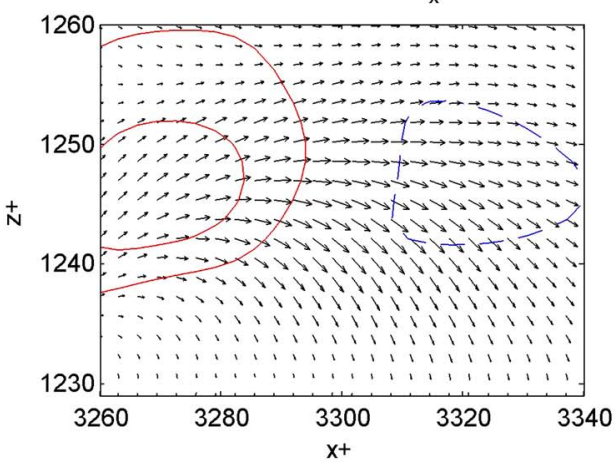

(d) contours of $v_{y}^{\prime}$

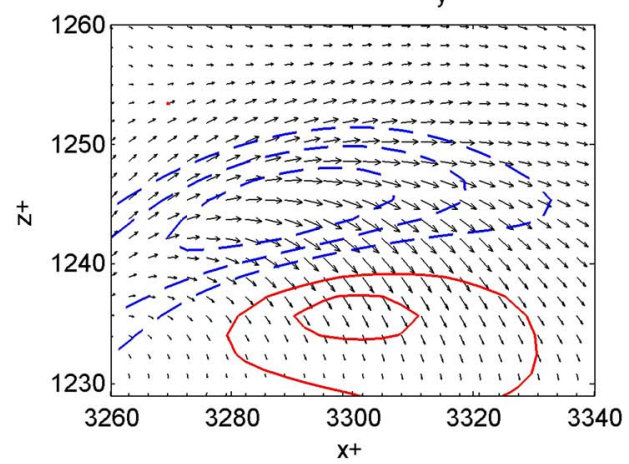

FIG. 16. Contours of (a) $-2 u_{y}^{\prime}\left(u_{x}^{\prime}+v_{y}^{\prime}\right)$, (b) $u_{y}^{\prime}$, (c) $u_{x}^{\prime}$, and (d) $v_{y}^{\prime}$. (a)-(d) A region in the plane $y^{+}=1$ of a snapshot. Dashed contours represent negative values; solid contours represent positive values. The contour level is 0.2 of the absolute maximum of the corresponding variable in the plane. The arrows represent the velocity vector $\left(u^{\prime}, w^{\prime}\right)$ in the plane.

stretches the shear layer in the $z$-direction $w_{z}^{\prime}>0$. This leads to generation of $u_{y}$ variance through $G_{121}+G_{122}$ (it has been verified that $G_{123}$ is also positive at $Y$, but smaller than $G_{121}+G_{122}$ ). Since the spanwise vorticity fluctuation is more or less equal to $-u_{y}^{\prime}$ at $Y$, the shear layer stretching is also stretching of spanwise vorticity. Thus this mechanism of fine-scale generation is likely to be the same as the stretching of spanwise vorticity fluctuation due to impingement of fluid from outer layers on the wall (splatting motion induced by streamwise vorticity). ${ }^{27,49}$ At the same location not only $u_{y}^{\prime}>0$, but also $\bar{u}_{y}>0$. As a consequence, $-2 \bar{u}_{y} u_{y}^{\prime}\left(u_{x}^{\prime}+v_{y}^{\prime}\right)>0$ at point $Y$, which means that this region also contributes to the production term $M^{(1)}$.

Finally, we will illustrate the shear layer stretching by a simple analytical solution of the Navier-Stokes equations in an infinite domain. We define

$$
\begin{aligned}
& u(x, y, z, t)=y e^{\zeta t}, \quad v(x, y, z, t)=-\zeta y, \quad w(x, y, z, t)=\zeta z \\
& p(x, y, z, t)=\frac{1}{2} \zeta\left(y^{2}-z^{2}\right) .
\end{aligned}
$$

By substitution this solution is easily verified as an exact solution of the incompressible NavierStokes equations. In fact this solution is a special case of the class of exact shear layer solutions, to which also Burgers Shear Layer Solution belongs, see Majda and Bertozzi (Section 1.6). ${ }^{37}$ Above solution expresses an exponential growth of the shear in $u$, due to compression of the shear layer in $y$-direction. In vortex stretching terms, the spanwise vorticity reduces to $-u_{y}$ and this vorticity is stretched by $w_{z}$. The equation of square shear is given by $\partial_{t}\left(u_{y}^{2}\right)=-2 u_{y}^{2} v_{y}=2 \zeta e^{2 \zeta t}$. This solution illustrates the local behavior of $u_{y}^{\prime}$ in the channel flow around point $Y$ of the snapshot shown. Quantitatively the velocity field is not exactly linear around $Y$, which means that in contrast to the 
exact solution shown above, viscous effects do play a role in the DNS; they balance or slow down the local growth of $u_{y}^{\prime}$ caused by the generation term.

\section{CONCLUSIONS}

Statistical profiles of the first- and second-order spatial derivatives of velocity and pressure were shown for turbulent channel flow at $R e_{\tau}=590$. The statistics were extracted from a high-resolution direct numerical simulation.

To quantify the anisotropic behavior of fine-scale structures, the variances of the derivatives were compared with the theoretical values for homogeneous isotropic turbulence. The centerline anisotropy coefficients of the contributions of the three velocity fluctuations to turbulence kinetic energy, turbulence dissipation, and destruction of turbulence dissipation were compared. The contributions to destruction were found to be more isotropic than the contributions to dissipation, which on their turn were more isotropic than the contributions to kinetic energy. However, the contributions to the generation of dissipation (the term with triple correlations of fluctuations of velocity derivatives) were found to be less isotropic than the contributions to dissipation. The anisotropic behavior of the velocity fluctuations at the centerline, which may not vanish for high Reynolds number, was attributed to turbulent transport. Directional viscous length scales $\mu_{i}$ based on firstand second-order derivatives were defined. These length scales were shown to be smaller and much more isotropic than the directional Taylor microscales $\lambda_{i}$. The high-Reynolds number limit of $\mu_{i}$ in isotropic turbulence was derived to be $3.3 \eta$. It appeared to be a reasonably accurate approximation for $\mu_{i}$ in the logarithmic and central regions of the channel flow at $R e_{\tau}=590$.

To quantify the non-Gaussian and intermittent behavior of fine-scale structures, higher-order moments and probability density functions of spatial derivatives were shown. The tails of some of these probability density functions of derivatives in turbulent channel flow were found to decay more slowly than both the Gaussian and the exponential distribution. Large skewnesses and flatnesses of derivatives were observed, in particular in the near wall region. The quantities were compared with DNS data of isotropic turbulence from literature. In the logarithmic and central regions, the intermittency (flatness) of each first-order derivative was found to be significantly larger than in isotropic turbulence at the same Taylor Reynolds number. Also the skewnesses of several derivatives showed anisotropic behavior in these regions.

Since the nine variances of first-order velocity derivatives are the distinct elements of the turbulence dissipation, the budgets of these nine variances were shown, together with the budget of the turbulence dissipation. The comparison of the budgets in the near-wall region indicated that the normal derivative of the fluctuating streamwise velocity $\left(\partial u^{\prime} / \partial y\right)$ plays a more important role than other components of the fluctuating velocity gradient. The small-scale generation term representing vortex and shear layer stretching caused by turbulent strain was analyzed in more detail; the distinct triple correlations of first-order velocity derivative fluctuations were compared with each other and the theoretical values in isotropic turbulence. Although this generation term is less isotropic than both the destruction term and the turbulence dissipation, a generalized velocity derivative skewness based on the same generation term is in the logarithmic and central regions quite close to the empirical value of derivative skewness in isotropic turbulence ( -0.5$)$. Finally, a typical mechanism of small-scale generation near the wall (around $y^{+}=1$ ) was observed: the intensification of positive $\partial u^{\prime} / \partial y$ by local strain fluctuation (compression in normal and stretching in spanwise direction).

Above conclusions on the statistics of spatial derivatives in turbulent channel flow have several implications. First, the profiles of the distinct first-order and second-order derivatives and the triple correlations that generate small scales provide more knowledge of quantities that play important roles in the long-standing problem whether solutions of the Navier-Stokes equations are regular (smooth) or not. ${ }^{37}$ Second, the budget of the turbulence dissipation rate may have implications for turbulence modeling. Although this budget was shown in previous works, ${ }^{25,27}$ this time the Reynolds number was higher, and for the first time source data of the profiles of a dissipation rate budget have been included into an internet DNS database of turbulent channel flow. Several physical implications of the present work are related to the conclusions on isotropy or anisotropy in turbulent channel flow. The approximately isotropic directional viscous length-scales and the increasing isotropy for 
increasingly fine-scale contributions to the velocity (first- and second-order derivatives summed per velocity component) in the logarithmic and central regions appear to confirm the Kolmogorov theory of local isotropy. In view of the confirmations of that theory, the conclusion that, away from the wall, the intermittencies of spatial derivatives are significantly higher than corresponding values mentioned in the literature of isotropic turbulence is surprising. Whether this conclusion also holds for $R e_{\tau}>590$ is an interesting question for future research.

\section{ACKNOWLEDGMENTS}

This work was sponsored by the Stichting Nationale Computerfaciliteiten (National Computing Facilities Foundation, NCF) for the use of supercomputer facilities, with financial support from the Nederlandse Organisatie voor Wetenschappelijk Onderzoek, NWO.

\section{APPENDIX: CENTERLINE STATISTICS}

Centerline values of various tensors discussed in the text are shown in Tables III-V. They are compared with the theoretical isotropic values presented in Sec. II. Table III shows the values for $A_{i j} / A$ and $A_{j}^{p} / A^{p}$, Table IV for $B_{i j k} / B$ and $B_{j k}^{p} / B^{p}$, and Table $\mathrm{V}$ for $G_{i j k} / G$.

TABLE III. Centerline values (C) of the variances of the first-order spatial derivatives of velocity and pressure compared to the theoretical isotropic values (I).

\begin{tabular}{|c|c|c|c|c|c|c|c|c|}
\hline \multirow[b]{2}{*}{$j$} & \multicolumn{2}{|c|}{$15 A_{1 j} / A$} & \multicolumn{2}{|c|}{$15 A_{2 j} / A$} & \multicolumn{2}{|c|}{$15 A_{3 j} / A$} & \multicolumn{2}{|c|}{$3 A_{j}^{p} / A^{p}$} \\
\hline & $\mathrm{C}$ & $\overline{\mathrm{I}}$ & $\mathrm{C}$ & $\overline{\mathrm{I}}$ & $\mathrm{C}$ & $\overline{\mathrm{I}}$ & $\mathrm{C}$ & $\overline{\mathrm{I}}$ \\
\hline 1 & 0.956 & 1 & 1.785 & 2 & 1.712 & 2 & 1.108 & 1 \\
\hline 2 & 2.528 & 2 & 1.021 & 1 & 2.143 & 2 & 1.047 & 1 \\
\hline 3 & 2.045 & 2 & 1.837 & 2 & 0.973 & 1 & 0.845 & 1 \\
\hline
\end{tabular}

TABLE IV. Centerline values (C) of the variances of the second-order spatial derivatives of velocity and pressure compared to the theoretical isotropic values (I).

\begin{tabular}{|c|c|c|c|c|c|c|c|c|c|}
\hline \multirow[b]{2}{*}{$j$} & \multirow[b]{2}{*}{$k$} & \multicolumn{2}{|c|}{$105 B_{1 j k} / B$} & \multicolumn{2}{|c|}{$105 B_{2 j k} / B$} & \multicolumn{2}{|c|}{$105 B_{3 j k} / B$} & \multicolumn{2}{|c|}{$15 B_{j k}^{p} / B^{p}$} \\
\hline & & $\mathrm{C}$ & $\overline{\mathrm{I}}$ & $\mathrm{C}$ & $\bar{I}$ & $\mathrm{C}$ & $\bar{I}$ & $\mathrm{C}$ & I \\
\hline 1 & 1 & 2.69 & 3 & 7.40 & 9 & 7.32 & 9 & 3.06 & 3 \\
\hline 2 & 2 & 11.24 & 9 & 3.36 & 3 & 10.84 & 9 & 3.25 & 3 \\
\hline 3 & 3 & 8.66 & 9 & 8.41 & 9 & 2.92 & 3 & 2.62 & 3 \\
\hline 1 & 2 & 2.17 & 2 & 1.94 & 2 & 3.07 & 3 & 1.09 & 1 \\
\hline 1 & 3 & 1.84 & 2 & 2.64 & 3 & 1.76 & 2 & 0.94 & 1 \\
\hline 2 & 3 & 3.34 & 3 & 2.07 & 2 & 2.21 & 2 & 1.00 & 1 \\
\hline
\end{tabular}

TABLE V. Centerline values (C) of the distinct contributions $G_{i j k}=-2 \overline{u_{i, j}^{\prime} u_{k, j}^{\prime} u_{i, k}^{\prime}}$ to the fine-scale generation term $G$, compared to the theoretical isotropic values (I).

\begin{tabular}{|c|c|c|c|c|c|c|c|}
\hline \multirow[b]{2}{*}{$i$} & \multirow[b]{2}{*}{$j$} & \multicolumn{2}{|c|}{$105 G_{i j 1} / G$} & \multicolumn{2}{|c|}{$105 G_{i j 2} / G$} & \multicolumn{2}{|c|}{$105 G_{i j 3} / G$} \\
\hline & & $\mathrm{C}$ & $\bar{I}$ & $\mathrm{C}$ & $\mathrm{I}$ & $\mathrm{C}$ & I \\
\hline 1 & 1 & 4.46 & 6 & 2.65 & $\frac{1}{2}$ & 1.25 & $\frac{1}{2}$ \\
\hline 1 & 2 & 2.98 & 4 & 8.47 & 4 & 5.88 & 6 \\
\hline 1 & 3 & 2.77 & 4 & 9.36 & 6 & 3.72 & 4 \\
\hline 2 & 1 & 1.10 & 4 & 5.97 & 4 & 4.73 & 6 \\
\hline 2 & 2 & -1.08 & $\frac{1}{2}$ & 7.78 & 6 & -1.04 & $\frac{1}{2}$ \\
\hline 2 & 3 & 4.25 & 6 & 6.11 & 4 & 1.67 & 4 \\
\hline 3 & 1 & 2.13 & 4 & 6.30 & 6 & 3.12 & 4 \\
\hline 3 & 2 & 7.06 & 6 & 6.88 & 4 & 2.38 & 4 \\
\hline 3 & 3 & 0.18 & $\frac{1}{2}$ & 1.65 & $\frac{1}{2}$ & 4.27 & 6 \\
\hline
\end{tabular}


${ }^{1}$ J. Kim, "Progress in pipe and channel flow turbulence, 1961-2011," J. Turbul. 13(45), 1-19 (2011).

2 J. Jiménez, "Near-wall turbulence," Phys. Fluids 25, 101302 (2013).

${ }^{3}$ J. Kim, P. Moin, and R. Moser, "Turbulence statistics in fully developed channel flow at low Reynolds number," J. Fluid Mech. 177, 133-166 (1987)

${ }^{4}$ N. D. Sandham and L. Kleiser, "The late stages of transition to turbulence in channel flow," J. Fluid Mech. 245, 319-348 (1992).

${ }^{5}$ R. D. Moser, J. Kim, and N. N. Mansour, “Direct numerical simulations of turbulent channel flow up to $\operatorname{Re}_{\tau}=590$," Phys. Fluids 11, 943-945 (1999).

${ }^{6} \mathrm{H}$. Abe, H. Kawamura, and Y. Matsuo, "Direct numerical simulation of a fully developed turbulent channel flow with respect to Reynolds number dependence," J. Fluids Eng. 123, 382-393 (2001).

${ }^{7}$ J. C. del Álamo and J. Jiménez, "Spectra of the very large anisotropic scales in turbulent channels," Phys. Fluids 15, L41-L43 (2003).

${ }^{8}$ Z. W. Hu, C. L. Morfey, and N. D. Sandham, "Wall pressure and shear stress spectra from direct numerical simulations of channel flow up to $\operatorname{Re} \tau=1440, "$ AIAA J. 44, 1541-1549 (2006).

${ }^{9}$ S. Hoyas and J. Jiménez, "Scaling of the velocity fluctuations in turbulent channels up to $R e_{\tau}=2003$," Phys. Fluids 18, 011702 (2006).

${ }^{10}$ S. Hoyas and J. Jiménez, "Reynolds number effects on the Reynolds-stress budgets in turbulent channels," Phys. Fluids 20, 101511 (2008).

${ }^{11}$ M. Kozuka, Y. Seki, and H. Kawamura, "DNS of turbulent heat transfer in a channel flow with a high spatial resolution," Int. J. Heat Fluid Flow 30, 514-524 (2009).

${ }^{12}$ P. E. Hamlington, D. Krasnov, T. Boeck, and J. Schumacher, "Local dissipation scales and energy dissipation-rate moments in channel flow," J. Fluid Mech. 701, 419-429 (2012).

${ }^{13}$ A. Lozano-Durán, O. Flores, and J. Jiménez, "The three-dimensional structure of momentum transfer in turbulent channels," J. Fluid Mech. 694, 100-130 (2012).

${ }^{14}$ P. Lenaers, Q. Li, G. Brethouwer, P. Schlatter, and R. Örlü, "Rare backflow and extreme wall-normal velocity fluctuations in near-wall turbulence,” Phys. Fluids 24, 035110 (2012).

${ }^{15}$ A. W. Vreman and J. G. M. Kuerten, "Comparison of direct numerical simulation databases of turbulent channel flow at $R e_{\tau}=180$," Phys. Fluids 26, 015102 (2014).

${ }^{16}$ T. A. Oliver, N. Malaya, R. Ulerich, and R. D. Moser, "Estimating uncertainties in statistics computed from direct numerical simulation," Phys. Fluids 26, 035101 (2014).

${ }^{17}$ M. Bernardini, S. Pirozolli, and P. Orlandi, "Velocity statistics in turbulent channel flow up to $R e_{\tau}=4000$," J. Fluid Mech. 742, 171-191 (2014).

${ }^{18}$ A. Lozano-Durán and J. Jiménez, "Effect of the computational domain on direct simulations of turbulent channels up to $R e_{\tau}=4200, "$ Phys. Fluids 26, 011702 (2014).

${ }^{19}$ H. M. Blackburn, N. N. Mansour, and B. J. Cantwell, “Topology of fine-scale motions in turbulent channel flow," J. Fluid Mech. 310, 269-292 (1996).

${ }^{20} \mathrm{~J}$. M. Wallace, "Twenty years of experimental and direct numerical simulation access to the velocity gradient tensor: What have we learned about turbulence?," Phys. Fluids 21, 021301 (2009).

${ }^{21}$ G. K. Batchelor and A. A. Townsend, "Decay of vorticity in isotropic turbulence," Proc. R. Soc. London, Ser. A 190, 534-555 (1947).

${ }^{22}$ G. K. Batchelor and A. A. Townsend, "The nature of turbulent motion at large wave-numbers," Proc. R. Soc. London, Ser. A 199, 238-255 (1949).

${ }^{23}$ A. A. Townsend, "On the fine-scale structure of turbulence," Proc. R. Soc. London, Ser. A 208, 534-542 (1951).

${ }^{24}$ R. A. Antonia, J. Kim, and L. W. B. Browne, "Some characteristics of small-scale turbulence in a turbulent duct flow," J. Fluid Mech. 233, 369-388 (1991).

${ }^{25}$ N. N. Mansour, J. Kim, and P. Moin, "Reynolds-stress and dissipation-rate budgets in turbulent channel flow," J. Fluid Mech. 194, 15-44 (1988).

${ }^{26}$ H. Tennekes and J. L. Lumley, A First Course in Turbulence (The MIT Press, Cambridge, MA, 1972).

${ }^{27}$ R. A. Antonia and J. Kim, "Low-Reynolds-number effects on near-wall turbulence," J. Fluid Mech. 276, 61-80 (1994).

${ }^{28}$ J. J. Gorski, J. M. Wallace, and P. S. Bernard, "The enstrophy equation budget of bounded turbulent shear flows," Phys. Fluids 6, 3197-3199 (1994).

${ }^{29}$ S. Tavoularis, J. C. Bennett, and S. Corrsin, "Velocity-derivative skewness in small Reynolds number, nearly isotropic turbulence," J. Fluid Mech. 88, 63-69 (1978).

${ }^{30} \mathrm{H}$. Ueda and J. O. Hinze, "Fine-structure turbulence in the wall region of a turbulent boundary layer," J. Fluid Mech. 67, 125-143 (1975).

${ }^{31}$ A. Vincent and M. Meneguzzi, "The spatial and statistical properties of homogeneous turbulence," J. Fluid Mech. 225, $1-20$ (1991).

${ }^{32}$ J. Jiménez, A. A. Wray, P. G. Saffman, and R. S. Rogallo, "The structure of intense vorticity in isotropic turbulence," J. Fluid Mech. 255, 65-90 (1993).

${ }^{33}$ A. Pumir, "A numerical study of pressure fluctuations in three-dimensional, incompressible, homogeneous, isotropic turbulence," Phys. Fluids 6, 2071-2083 (1994).

${ }^{34} \mathrm{P}$. Vedula and P. K. Yeung, "Similarity scaling of acceleration and pressure statistics in numerical simulations of isotropic turbulence," Phys. Fluids 11, 1208-1220 (1999).

${ }^{35}$ N. Cao, S. Chen, and G. D. Doolen, "Statistics and structures of pressure in isotropic turbulence," Phys. Fluids 11 2235-2250 (1999).

${ }^{36}$ T. Ishihara, Y. Kaneda, M. Yokokawa, K. Itakura, and A. Uno, "Small-scale statistics in high-resolution direct numerical simulation of turbulence: Reynolds number dependence of one-point velocity gradient statistics," J. Fluid Mech. 592, 335-366 (2007) 
${ }^{37}$ A. J. Majda and A. L. Bertozzi, Vorticity and Incompressible Flow (Cambridge University Press, Cambridge, 2002).

${ }^{38}$ P. Bradshaw and B. Perot, "A note on turbulent energy dissipation in the viscous wall region," Phys. Fluids A 5, 3305-3306 (1993).

${ }^{39}$ G. K. Batchelor, The Theory of Homogeneous Turbulence (Cambridge University Press, Cambridge, 1960).

${ }^{40}$ T. de Kármán and L. Howarth, "On the statistical theory of isotropic turbulence," Proc. R. Soc. London, Ser. A 164, 192-214 (1938).

${ }^{41}$ S. B. Pope, Turbulent Flows (Cambridge University Press, Cambridge, 2000).

${ }^{42}$ J. G. M. Kuerten, C. W. M. van der Geld, and B. J. Geurts, "Turbulence modification and heat transfer enhancement by inertial particles in turbulent channel flow," Phys. Fluids 23, 123301 (2011).

${ }^{43}$ B. J. Geurts and J. G. M. Kuerten, "Ideal stochastic forcing for the motion of particles in large-eddy simulation extracted from direct numerical simulation of turbulent channel flow," Phys. Fluids 24, 081702 (2012).

${ }^{44}$ P. R. Spalart, R. D. Moser, and M. M. Rogers, "Spectral methods for the Navier-Stokes equations with one infinite and two periodic directions," J. Comput. Phys. 96, 297-324 (1991).

${ }^{45}$ S. G. Saddoughi and S. V. Veeravalli, "Local isotropy in turbulent boundary layers at high Reynolds number," J. Fluid Mech. 268, 333-372 (1994).

${ }^{46}$ J. Jiménez, "How linear is wall-bounded turbulence?," Phys. Fluids 25, 110814 (2013).

${ }^{47}$ Y. Kaneda, T. Ishihara, M. Yokokawa, K. Itakura, and A. Uno, "Energy dissipation rate and energy spectrum in high resolution direct numerical simulations of turbulence in a periodic box," Phys. Fluids 15, L21-L24 (2003).

${ }^{48}$ V. Yakhot, "Pressure-velocity correlations and scaling exponents in turbulence," J. Fluid Mech. 495, 135-143 (2003).

${ }^{49}$ P. Moin and J. Kim, "Numerical investigation of turbulent channel flow," J. Fluid Mech. 118, 341-377 (1982). 